NBER WORKING PAPER SERIES

COMPARATIVE STATICS IN MARKETS FOR INDIVISIBLE GOODS

\author{
Andrew Caplin \\ John V. Leahy \\ Working Paper 16285 \\ http://www.nber.org/papers/w16285
}

NATIONAL BUREAU OF ECONOMIC RESEARCH
1050 Massachusetts Avenue
Cambridge, MA 02138
August 2010

We thank Mamoru Kaneko, John Leahy Sr., Jeffrey Mensch, Victor Norman, Michael Reiter, and Ennio Stacchetti, for helpful comments. Leahy thanks the NSF for financial support. The views expressed herein are those of the authors and do not necessarily reflect the views of the National Bureau of Economic Research.

NBER working papers are circulated for discussion and comment purposes. They have not been peerreviewed or been subject to the review by the NBER Board of Directors that accompanies official NBER publications.

(C) 2010 by Andrew Caplin and John V. Leahy. All rights reserved. Short sections of text, not to exceed two paragraphs, may be quoted without explicit permission provided that full credit, including $\odot$ notice, is given to the source. 
Comparative Statics in Markets for Indivisible Goods

Andrew Caplin and John V. Leahy

NBER Working Paper No. 16285

August 2010

JEL No. C63,D40,E1,R31

\begin{abstract}
$\underline{\text { ABSTRACT }}$
We complete the study of comparative statics initiated in Caplin and Leahy [2010], which introduced a new mathematical apparatus for understanding NTU allocation markets, as such covering the housing market and other markets for large indivisible goods. We introduce homotopy methods to characterize how equilibrium changes in response to arbitrary parameter changes. Generically, we show that there can be five and only five qualitatively distinct forms of market transition: Graft; Prune and Plant; Prune and Graft; Cycle and Reverse; and Shift and Replant. Our path-following methods identify new algorithms for computing market equilibria.
\end{abstract}

\author{
Andrew Caplin \\ Department of Economics \\ New York University \\ 19 W. 4th Street, 6th Floor \\ New York, NY 10012 \\ and NBER \\ andrew.caplin@nyu.edu \\ John V. Leahy \\ Department of Economics \\ New York University \\ 19 W. 4th Street, 6th Floor \\ New York, NY 10012 \\ and NBER \\ john.leahy@nyu.edu
}




\title{
Comparative Statics in Markets for Indivisible Goods
}

\author{
Andrew Caplin and John Leahy* \\ New York University and N.B.E.R.
}

August 2010

\begin{abstract}
This paper builds upon Caplin and Leahy [2010], which introduced a new mathematical apparatus for understanding NTU allocation markets, as such covering the housing market and other markets for large indivisible goods. In the current paper we complete the study of comparative statics initiated therein. We introduce homotopy methods to characterize how equilibrium changes in response to arbitrary parameter changes. Generically, we show that there can be five and only five qualitatively distinct forms of market transition: Graft; Prune and Plant; Prune and Graft; Cycle and Reverse; and Shift and Replant. Our pathfollowing methods identify new algorithms for computing market equilibria.
\end{abstract}

\section{Introduction}

Among the most significant of all markets are those, such as the housing market, that allocate large indivisible goods to households. These goods are best modeled as allocation markets with non-transferable utility (NTU). Caplin and Leahy [2010] (henceforth CL) introduced a new mathematical apparatus for understanding these markets. In the current paper we complete the study of comparative statics initiated therein. We use homotopy methods to characterize how the minimum price competitive equilibrium changes in response to arbitrary parameter changes. Given an initial equilibrium, we follow a path through parameter space, building up the discrete change in equilibrium from infinitesimal ones. We show that along the generic path equilibria evolve in a very controlled manner. There are five and only five qualitatively distinct forms of

*We thank Mamoru Kaneko, John Leahy Sr., Jeffrey Mensch, Victor Norman, Michael Reiter, and Ennio Stacchetti, for helpful comments. Leahy thanks the NSF for financial support. 
market transition. The homotopy path decomposes all comparative statics into these five basic operations.

In addition to identifying market transitions, we show that our path-following methods are ideally suited to algorithmic use. They can be used in principle to compute minimum price equilibria from a starting point with a trivial equilibrium. The resulting algorithms may help to overcome computational barriers to application of the NTU allocation model. ${ }^{1}$ It is also not hard to envision adopting our methods to studying the dynamic properties of markets for indivisible goods by recasting the homotopy paths as sample paths which characterize the evolution of the market over time.

Demange and Gale [1985] show that there exists a minimum price equilibrium in the class of allocation markets that we consider, those in which each agent is either a buyer or a seller. CL showed that these equilibria correspond to the solutions to a certain optimization problem. This optimization is over a set of mathematical structures, called GA-structures, that combine an allocation of goods to buyers along with a particular class of directed graphs that summarize key indifference relations among goods. CL show for small changes in parameter values the allocation and the indifference relations that characterize the minimum price competitive equilibrium are unlikely to change. Given that "local" comparative statics involve a fixed GA-structure, they are able to show that changes in model parameters that cause the price of a given good to change impact only those goods that "follow" it in the corresponding directed graph.

In this paper we use GA-structures to characterize comparative statics in the large. We study how the allocation of goods and the pattern of local interdependence changes when there is a discrete shift in the character of the minimum price equilibrium. While there are examples in which the most minor change in parameters cause the entire structure of the equilibrium to change in arbitrary ways, we show such cases to be the exception rather than the rule. We introduce a natural definition of regularity for comparative static paths and show generically small parameter changes can induce five and only five distinct forms of market transitions. We use GA-structures to illustrate the precise nature of each such transition. For reasons that will be clear, the market transitions are labeled as: Grafting; Pruning; Prune and Graft; Cyclic Reversal; and Shift and Re-plant.

Markets for indivisible goods are characterized by multiple equilibria. Due to the presence of non-convexities, small changes in prices often do not alter the equilibrium allocation. We focus

\footnotetext{
${ }^{1}$ The transferable utility case is well covered in this regard: the Hungarian algorithm of Kuhn [1955] and Munkres [1957] can be used to compute the equilibrium allocation, while the ascending auction mechanism of Demange, Gale and Sotomayor (1986) solves for the minimum price equilibrium in a discretized version of the model.
} 
on the minimum equilibrium price for a number of reasons. First, we learn about the entire range of possible equilibria. The minimum and maximal equilibrium prices define the boundaries of all equilibria. The two prices have the same structure. The maximal equilibrium price is simply the dual of the minimal, in which the roles of buyers and sellers are reversed. Moreover, CL show that all equilibrium prices may be thought of as minimal equilibrium prices for some set of parameter values. Finally, Demange and Gale [1985] show that the minimal equilibrium price vector is not manipulable by buyers, making it a natural benchmark for multi-unit auctions.

Section 2 presents the general model and summarizes relevant results from CL. The additional results in this paper rest in part on our ability to count the number of distinct GA-structures. The key cardinality results are in section 3. They are derived by connecting the number of GA-structures with the structure of market demand at minimum equilibrium prices. We show that there is typically only one GA-structure, and that the most important points of market transition involve two and only two such structures. Section 4 introduces the domain in which we study comparative statics, which involves paths through a rich space of model parameters. Section 5 identifies the sense in which comparative static transitions are almost always "regular", in that the replacement for a given GA-structure is a unique second element that appears at a point of transition. Section 6 identifies the five generic forms of market adjustment. Section 7 shows how to use path-following methods to algorithmically identify the minimum equilibrium price. Section 8 concludes. All proofs are in the Appendix.

\section{Background}

\subsection{Model}

There is a set of buyers $x_{a} \in X, 1 \leq a \leq m$, and a set of indivisible goods $y_{i} \in Y, 1 \leq i \leq n$ with $n>m$. The goods are initially held by the sellers. Buyers may purchase the indivisible goods from sellers by making a transfer in terms of a homogeneous, perfectly divisible, numeraire good, which may be thought of as money.

Buyers derive utility from at most one element of $Y$. The payoff for buyer $x_{a}$ is summarized by the utility function $U_{a}: Y \times \mathbb{R} \rightarrow \mathbb{R}$, where $U_{a}\left(y_{i}, p_{i}\right)$ is the utility to $x_{a}$ from the purchase of $y_{i}$ with a transfer of $p_{i}$ of the numeraire good.

The supply side is trivial. Sellers choose only whether or not to sell. They do not purchase the indivisible goods from other sellers. Each seller wishes only to obtain the highest possible price above a reservation level. Let $r \in \mathbb{R}_{+}^{n}$ denote the vector of seller reservation prices. Overall, 
we can limit attention to a set $\Pi$ in the search for competitive equilibria,

$$
\Pi=\left\{p \in \mathbb{R}_{+}^{n} \mid p \geq r \text { and } p_{n-a}=r_{n-a}=0 \text { all } 0 \leq a \leq m-1\right\}
$$

Given any price vector $p \in \Pi$, the (non-empty) demand correspondence $D_{a}(p)$ specifies members of $Y$ that maximize utility the utility of buyer $x_{a}$,

$$
D_{a}(p)=\left\{y_{i} \in Y \mid U_{a}\left(y_{i}, p_{i}\right) \geq U_{a}\left(y_{k}, p_{k}\right) \text { for all } y_{k} \in Y_{a}\right\}
$$

An allocation $\mu: X \rightarrow Y$ is a one-to-one mapping from buyers to goods. The set of all allocations is $M$

A competitive equilibrium is a price vector and an allocation such that all buyers choose optimally and all goods with prices above their reservation level are allocated.

Definition A competitive equilibrium is a pair $\left(p^{*}, \mu^{*}\right)$ with $p^{*} \in \mathbb{R}^{n}$ and $\mu^{*} \in M$ such that:

1. $\mu_{a}^{*} \in D_{a}\left(p^{*}\right)$ for all $x_{a} \in X$.

2. $p_{i}^{*} \geq r_{i}$ for all $y_{i} \in Y$.

3. If $y_{i} \in U\left(p^{*}\right)$, then there exists $x_{a} \in X$ such that $\mu_{a}^{*}=y_{i}$.

The first condition is buyer optimality. The allocation must maximize the utility of each buyer. The second condition is seller optimality. No seller will part for a good for less than the reservation price. The third states that all goods with prices above reservation must be allocated. This ensures that supply is equal to demand.

We make continuity and range assumptions that guarantee that the set of equilibria is a closed lattice (see Demange and Gale [1985]). The first assumption is a straight forward regularity assumption. The second assumption in combination with the first ensures that given any buyer, any two goods, and a price for one of the goods, there is a price for the second that makes the buyer indifferent between the two goods.

Assumption A For each buyer $x_{a} \in X$ and good $y_{i} \in Y$,

1. $U_{a}\left(y_{i}, p_{i}\right)$ is continuously differentiable in $p_{i}$ and strictly decreasing in $p_{i}$.

2. $\lim _{p_{i} \rightarrow \infty} U_{a}\left(y_{i}, p_{i}\right)=-\infty$ and $\lim _{p_{i} \rightarrow-\infty} U_{a}\left(y_{i}, p_{i}\right)=\infty$. 
With Assumption A we know not only that there is a competitive equilibrium allocation, but also that there is a minimum equilibrium price (henceforth MEP) $\underline{p} \in \Pi$, which has the property that it is an equilibrium price and that, for any equilibrium price,

$$
p_{i} \geq \underline{p}_{i} \text { all } y_{i} \in Y
$$

Note we have presented the model without explicit reference to buyers' endowment of the numeraire good. We have implicitly assumed that buyers hold enough of the numeraire good to buy the goods that they are assigned at the competitive equilibrium prices. An alternative approach would have been to have expressed utility in terms of the endowment left over after purchase and to have included the additional assumption that each buyer preferred exit to spending all of his endowment on a purchase. This condition would hold for any utility function that satisfied the Inada conditions. This is the approach taken by Kaneko (1982) and is equivalent to the current one.

\subsection{GA-Structures}

CL introduce GA-structures, which combine an allocation of goods with a particular class of graph, to study the structure of equilibria in the allocation model. The graphs in question are directed graphs on vertex set $Y$ that: are forests of trees (i.e. contain no cycles); that are rooted, in the sense that each component tree has a unique element that is specified as its root, and in which each edge is directed away from the root. We let $\mathcal{F}$ denote the class of directed rooted forests on vertex set $Y$. Note the insistence that all goods that are at the "tail" of a directed edge are allocated.

Definition A graph-allocation structure (GA-structure) comprises:

1. A directed graph $F=(Y, R, E)$ with vertex set $Y$, root set $R \subseteq Y$, and edge set $E \in Y^{2}$ in which $F$ is a forest of trees, each component of $F$ contains a unique element of $R$ and each edge $\left(y_{i}, y_{k}\right) \in E$ is directed away from the corresponding element of $R$.

2. An allocation $\mu \in M$ such that, if $\left(y_{i}, y_{k}\right) \in E$, then there exists $x_{a} \in X$ such that $\mu\left(x_{a}\right)=y_{i}$.

We let $\mathcal{G}$ denote the set of all GA-structures. 
CL construct a natural mapping from $\mathcal{G}$ into prices by first pricing root goods at reservation, and then iteratively pricing successors by indifference of the individual allocated to their unique predecessor. Assumption A guarantees that this construction is well-defined.

Definition The function $q: \mathcal{G} \rightarrow \mathbb{R}^{n}$, the price generated by graph-allocation structure $(\mu, F) \in$ $\mathcal{G}$ is defined by setting $q_{i}(\mu, F)=r_{i}$ for all $y_{i} \in R(F) \equiv A_{0}$, with iterative pricing of successor goods $y_{k} \in S$ of $A_{s}$ for $s \geq 1$ based on the indifference condition,

$$
U_{a}\left(y_{i}, q_{i}(\mu, F)\right)=U_{a}\left(y_{k}, q_{k}(\mu, F)\right) .
$$

where $\mu_{a}=y_{i}$, and $\left(y_{i}, y_{k}\right) \in E(F)$, so that $y_{i} \in A_{s}$ is the direct predecessor of $y_{k} \in S$.

\subsection{The Min-Max Theorem}

CL establish that with Assumption A, a solution to an optimization problem on these generated prices identifies the minimum equilibrium price. It also establishes that any allocation that forms part of a minimum price equilibrium (MPE) must also be identifiable from this optimization problem.

Theorem (CL): $q\left(\mu^{*}, F^{*}\right)$ is a minimum equilibrium price if and only if:

$$
\sum_{i \in\{1 \ldots n\}} q_{i}\left(\mu^{*}, F^{*}\right)=\min _{\mu \in M} \max _{F \in\{F \in \mathcal{F} \mid(\mu, F) \in \mathcal{G}\}} \sum_{i \in\{1 \ldots n\}} q_{i}(\mu, F) .
$$

Moreover, let $\left(\mu^{*}, F^{*}\right)$ be an argument that solves $(2.2)$, if $q_{i}\left(\mu^{*}, F^{*}\right)>r_{i}$ for all $y_{i} \notin R(F)$ then $\left(\mu^{*}, q_{i}\left(\mu^{*}, F^{*}\right)\right)$ is a MPE.

Comparative statics are particularly simple when there is one and only one element $\left(\mu^{*}, F^{*}\right) \in$ $\mathcal{G}$ that solves (2.2) for some range of parameters. In such cases, a continuous change in the model's parameters do not change in the allocation $\mu$ or the graph $F$. Changes in sellers reservation have a direct effect on prices of root goods and changes in a buyer utility directly affects the prices of goods that are direct successors of the good that that buyer is allocated to. These changes propagate through the graph structure affecting the price of all successors in $F$.

\section{Cardinality and the Demand Graph}

The focus of this paper is on cases in which a change in parameters is large enough to force a change in the equilibrium GA-structure. It turns out that this generally happens in a very 
controlled manner. There is a sense in which it is rare to come across more than two solutions to (2.2). For most parameter values there is only one solution. As one moves through the parameter space, the generic path follows this single solution until at some point there a second solution appears along with the first. ${ }^{2}$ The path then follows one of these two solutions for a while. The current section formalizes these arguments using counting arguments.

\subsection{The GAME set}

We begin by stating exactly what we mean to count. It is the set of $(\mu, F) \in \mathcal{G}$ which generate the minimum equilibrium price and also have the property that $\mu$ allocates buyers not only to the tails but to the heads of all edges in $E(F) .^{3}$

Definition: The graph allocation minimum price equilibrium (GAME) set is,

$$
\Phi=\left\{(\mu, F) \in \mathcal{G} \mid(\mu, q(\mu, F)) \text { is a MPE and for all }\left(y_{i}, y_{k}\right) \in E(F) \Longrightarrow \mu_{a}=y_{k} \text { some } x_{a} \in X\right\}
$$

The condition that $\mu$ allocates a buyer to the head of each edge in $E(F)$ rules out some uninteresting cases. Suppose, for example, that two goods $y_{1}$ and $y_{2}$ are both priced at reservation, and that there is only one buyer who is indifferent between them at these prices. In this case, there are four elements of $\mathcal{G}$ that generate the minimum price competitive equilibrium and hence "solve" the min-max problem. There are two potential allocations, and for each allocation we may either price both goods at reservation or use the buyer's indifference to price the unallocated good. The condition that $\mu$ allocates a buyer to the head of each edge in $E(F)$ rules out the use of indifference to price the unallocated good. In this case, $\Phi=2$.

Note that $\Phi$ depends on the parameters of the model through the minimum price equilibrium. We suppress this dependence, as we are considering a fixed set of parameters at this point.

\subsection{Demand Graphs and Condition $M$}

The cardinality of the GAME set $\Phi$ is basic in the work that follows. We derive conditions under which $\Phi$ is unique and under which it has two elements. We develop these cardinality results using properties of demand at minimum equilibrium prices. We introduce the MPE

\footnotetext{
${ }^{2}$ Throughout this paper we use the term generic in the topological sense. A property is generic if it holds on a dense open set.

${ }^{3}$ The definition of $\Phi$ in this paper differs from that in CL. In that paper we did not need the restriction that the heads of all edges are allocated.
} 
demand graph and a class of its subgraphs that turn out to be of particular importance in this characterization.

Definition: The MPE demand graph $D(\underline{p})=\left(X, Y, \mathcal{E}^{*}\right)$ is a bipartite graph with partition $X$ and $Y$, and an edge set defined by,

$$
\mathcal{E}^{*}=\left\{\left(x_{a}, y_{j}\right) \in X \times Y \mid y_{j} \in D_{a}(\underline{p})\right\}
$$

Definition: The edge set $\mathcal{E} \subset \mathcal{E}^{*}$ is said to satisfy Condition $M$, written $\mathcal{E} \in \mathcal{M}(\underline{p})$, if there exists a partition $\left\{Y_{l}^{\mathcal{E}} \mid 0 \leq l \leq L\right\}$ of $Y$ such that,

M1. $Y_{0}^{\mathcal{E}}$ is comprised of the elements of $Y$ that isolated in the demand graph.

M2. For $l \geq 1$, each element of $Y_{l}^{\mathcal{E}}$ is connected to the other elements of $Y_{l}^{\mathcal{E}}$, but not to elements of $Y \backslash Y_{l}^{\mathcal{E}}$.

M3. For $l \geq 1$, there is one and only one element, $\sigma_{l}^{\mathcal{E}} \in Y$, of degree (valence) 1 in each partition set $Y_{l}^{\mathcal{E}}$. All other elements of $Y_{l}^{\mathcal{E}}$ have degree 2.

M4. For $l \geq 1,\left|X_{l}^{\mathcal{E}}\right|=\left|Y_{l}^{\mathcal{E}}\right|$ where $X_{l}^{\mathcal{E}}=\left\{x_{a} \in X \mid\left(x_{a}, y_{i}\right) \in \mathcal{E}\right.$ some $\left.y_{i} \in Y_{l}^{\mathcal{E}}\right\}$.

M5. $\underline{p}_{i}>r_{i}$ implies that the degree of $y_{i}$ is equal to two.

Both the demand graph $D$ and the set of subgraphs $\mathcal{M}$ depend on the price vector $\underline{p}$ which determines the set of goods in each buyer's demand set. To save on notation, we suppress this dependence in much of what follows as we are considering a fixed set of parameters at this point.

\subsection{A Counting Lemma}

The reason for introducing these particular graphs is that they will correspond in a precise manner with elements of $\Phi . \mathcal{M}$ and $\Phi$ are essentially equivalent. Given a MEP $\underline{p}$, we introduce a bijection $\eta: \mathcal{M} \rightarrow \Phi$ between $\Phi$ and $\mathcal{M}$ that provides structure to counting arguments. The proofs of all propositions are in the appendix.

Lemma 1: Let $\underline{p}$ be a minimum equilibrium price vector. There exists a bijection $\eta: \mathcal{M} \rightarrow \Phi$ so that

$$
|\Phi|=|\mathcal{M}|
$$


Given $\mathcal{E} \in \mathcal{M}$, the graph $(X, Y, \mathcal{E})$ is a bipartite graph with partition $\{X, Y\}$. Since it is a selection from the demand graph at $\underline{p}$, each buyer is matched only to goods that are optimal at these prices. With conditions M1-M4, $(X, Y, \mathcal{E})$ is acyclic. Given one good in each component with degree 1, a cycle would either lead to fewer buyers than sellers or require a good with degree greater than two. Each partition element $Y_{l}^{\mathcal{E}}$ for $l \geq 1$ therefore corresponds to a distinct tree. In each such tree, the root good has degree 1 and all others have degree 2. F is constructed recursively, beginning with the valence one goods and then at each step adding as direct successors all goods that are two edges distant in $\mathcal{E}$. Finally, $Y_{0}^{\mathcal{E}}$ is added to the root set. The equality between the number of goods and buyers in each such set generates a natural mapping $\mu$. The valence one goods are allocated to their neighbors in $\mathcal{E}$. Removing these buyers creates a new set of valence one goods, who are then allocated to their neighbors, and so on. Note that the equality of buyers and sellers in M4 guarantees that all goods in $Y_{l}^{\mathcal{E}}$ are allocated. This implies that all non-root goods are allocated and that the heads and tails of all edges in $E(F)$ are allocated. Finally, it is easy to show that the $(\mu, F)$ generated in this way generates $\underline{p}$, so that $(\mu, F) \in \Phi$.

This bijection is illustrated in Figure 1 for a case in which there are five buyers and six goods. Figure 1(a) illustrates $\mathcal{E} \in \mathcal{M}$, with the partition $\left\{Y_{l}^{\mathcal{E}} \mid 0 \leq l \leq L\right\}$. The sixth good is unassigned, $Y_{0}^{\mathcal{E}}=y_{6}$. The other two partition sets are $Y_{1}^{\mathcal{E}}=\left\{y_{1}, y_{2}, y_{3}\right\}$ and $Y_{2}^{\mathcal{E}}=\left\{y_{4}, y_{5}\right\}$. To draw the corresponding allocation $\eta(\mathcal{E})$ we iterate from the goods of degree 1 , so that $\mu_{i}=y_{i}$ for $1 \leq i \leq 5$. The forest is found by placing down the three root goods, $y_{1}, y_{4}$, and $y_{6}$ (which is unoccupied) and then drawing all edges corresponding to additional demands of their matched buyers, $x_{1}$ and $x_{4}$. Buyer $x_{4}$ demands $y_{5}$ in addition to $y_{4}$, while buyer $x_{1}$ demands $y_{2}$ and $y_{3}$ in addition to $y_{1}$. Adding these as directed edges from the respective root good completes the forest, as illustrated in Figure 1(b). The inverse mapping is also clear: if one starts from the forest of directed rooted trees, all goods are demanded by the individual allocated to them, and any individual allocated to a good at the origin of a directed edge demands all goods to which such edges are directed.

\subsection{Uniqueness}

A characterization of demand graphs that give rise to uniqueness of the GAME set immediately from Lemma 1. In the statement, $d^{*}\left(y_{i}\right)$ is the degree of vertex $y_{i}$ associated with demand graph $D$.

Corollary 1: $\mathcal{E}^{*} \in \mathcal{M}$ and $d^{*}\left(y_{i}\right)=2 \Longrightarrow \underline{p}_{i}>r_{i}$ then $|\Phi|=1$. 
Since $\mathcal{E}^{*} \in \mathcal{M}$ all goods have valence less than two. Any other $\mathcal{E} \in \mathcal{M}$ is a subgraph of $\mathcal{E}^{*}$, and therefore involves reducing the valence of some good. Reducing $d^{*}\left(y_{i}\right)$ from two to one violates M5 since $d^{*}\left(y_{i}\right)=2 \Longrightarrow \underline{p}_{i}>r_{i}$. Reducing $d^{*}\left(y_{i}\right)$ from one to zero reduces the number of goods that are connected to buyers and violates M4.

We identify $\mathcal{U} \subset \mathcal{M}$ as the set of graphs satisfying the conditions of the uniqueness theorem. This set is of particular value in enumerating elements of $\Phi$ when there is more than one.

\subsection{Cardinality Two}

Corollary 1 implies that the existence of multiple equilibrium GA structures is associated either with one or more "extra" indifference relationships in the demand graph that can be removed and have an edge structure that satisfies Condition M, or with some set of goods with valence greater than one which have prices equal to their reservation values.

Our next result concerns circumstances in which $|\Phi|=2$. This is the case whenever the demand graph associated with the minimum price equilibrium has one and only one edge more than some subgraph $\tilde{\mathcal{E}} \subset \mathcal{E}^{*}$ such that $\tilde{\mathcal{E}} \in \mathcal{U}$.

While the theorem is proven in the appendix in standard analytic manner, certain of the arguments are best understood in the figures that follow.

Theorem 1: $|\Phi|=2$ if and only if there exists $(\bar{x}, \bar{y}) \in \mathcal{E}^{*}$ such that $\mathcal{E}^{*} /(\bar{x}, \bar{y}) \equiv \tilde{\mathcal{E}} \in \mathcal{U}$.

The proof considers five cases. Each case is distinguished by the position of the good $\bar{y}$ and the buyer $\bar{x}$. We illustrate how beginning with $\tilde{\mathcal{E}} \in \mathcal{U}$, the addition of an extra edge makes possible the construction of a second element $\mathcal{E}^{\prime} \in \mathcal{M}$.

Figure 2 illustrates a case in which $\bar{y} \in Y_{0}^{\tilde{\varepsilon}}$. In this example, there are two buyers and three goods. Figure 2(a) illustrates the edge set $\tilde{\mathcal{E}} \in \mathcal{U}$ :

$$
\tilde{\mathcal{E}}=\left\{\left(x_{1}, y_{1}\right),\left(x_{1}, y_{2}\right),\left(x_{2}, y_{2}\right)\right\}
$$

The additional edge in $\mathcal{E}^{*}$ is $\left(x_{2}, y_{3}\right)$ as illustrated in Figure 2(b). We obtain a second element of $\mathcal{E}^{\prime} \in \mathcal{M}$ by removing the edge $\left(x_{1}, y_{1}\right)$, as illustrated in figure $2(\mathrm{c})$.

Figure 3 illustrates a case in which $\bar{y} \in Y_{l}^{\tilde{\mathcal{E}}}$ and $\bar{x} \in X_{l}^{\tilde{\mathcal{E}}}$ for some $l \geq 1$. In this example, there are three buyers and three goods. We begin with the edge set $\tilde{\mathcal{E}} \in \mathcal{U}$ comprising,

$$
\tilde{\mathcal{E}}=\left\{\left(x_{1}, y_{1}\right),\left(x_{1}, y_{2}\right),\left(x_{2}, y_{2}\right),\left(x_{1}, y_{3}\right),\left(x_{3}, y_{3}\right)\right\},
$$


This is illustrated in Figure 3(a). The additional edge in $\mathcal{E}^{*}$ is $\left(x_{3}, y_{2}\right)$ as illustrated in Figure $3(\mathrm{~b}) . \mathcal{E}^{\prime} \in \mathcal{M}$ is obtained by removing edge $\left(x_{1}, y_{2}\right)$, as illustrated in Figure $3(\mathrm{c})$.

Figure 4 illustrates a case with $\bar{\mu} \in Y_{l}^{\tilde{\mathcal{E}}}$ and $\bar{y} \in Y_{m}^{\tilde{\mathcal{E}}}$ with $m \notin\{0, l\}$, and with $\bar{y}$ of valence 2 in $\tilde{\mathcal{E}}$. Again there are three buyers and three goods. The edge set $\tilde{\mathcal{E}} \in \mathcal{U}$ comprises,

$$
\tilde{\mathcal{E}}=\left\{\left(x_{1}, y_{1}\right),\left(x_{2}, y_{2}\right),\left(x_{2}, y_{3}\right),\left(x_{3}, y_{3}\right)\right\}
$$

as illustrated in Figure 4(a). The additional edge in $\mathcal{E}^{*}$ is $\left(x_{1}, y_{3}\right)$ as illustrated in Figure $4(\mathrm{~b})$. $\mathcal{E}^{\prime} \in \mathcal{M}$ is obtained by removing $\left(x_{2}, y_{3}\right)$, as illustrated in figure $4(\mathrm{c})$.

In the final case, $\bar{\mu} \in Y_{l}^{\tilde{\mathcal{E}}}, \bar{y} \in Y_{m}^{\tilde{\mathcal{E}}}$ with $m \notin\{0, l\}$, and $\bar{y}$ has valence 1 . This case is similar to that in Figure 4, except that $\left(x_{2}, y_{3}\right) \notin \tilde{\mathcal{E}}$.

It is important to note that there is something special about the addition of a single edge to $\tilde{\mathcal{E}} \in \mathcal{U}$ that leads to one and only one additional element of $\Phi$. The addition of two edges does not have a simple structure. It is easy to construct examples in which the addition of two edges to $\tilde{\mathcal{E}} \in \mathcal{U}$ can lead to $\Phi$ having three, four, or six elements depending on the position of the additional goods and buyers in the graph $(X, Y, \tilde{\mathcal{E}})$.

\section{Regular Comparative Static Paths}

We now consider a set of models indexed by a parameter vector $\lambda \in \Lambda$ and consider comparative statics with respect to $\lambda$. CL show that if the $q(\mu, F ; \lambda)$ are smooth in a sense to be defined below, the effect of an infinitesimal change in $\lambda$ on the minimum equilibrium price can almost always be analyzed using a fixed GA structure. In this case, the change in $\lambda$ only affects the prices of goods directly influenced by the change in $\lambda$ and their successors in the relevant graph $F$.

Discrete changes in $\lambda$ are more complicated because they lead to shifts in the GA structure. In this section, we use homotopy methods to characterize discrete comparative statics. ${ }^{4}$ Given two points $\lambda_{0}$ and $\lambda_{1}$ in $\Lambda$, we start with a known equilibrium at $\lambda_{0}$ and consider paths through $\Lambda$ running from $\lambda_{0}$ to $\lambda_{1}$. We let $\Phi(\lambda)$ denote the dependence of the GAME set on $\lambda$. We show that if the $q(\mu, F ; \lambda)$ are smooth and if $\Lambda$ is large enough, then $\Phi(\lambda)$ has the following two properties along the generic path: (1) $\Phi(\lambda)$ has at most two elements at every point along the path; and (2) $\Phi(\lambda)$ has two elements at a only finite number of points so that it is single valued almost everywhere. We call these paths regular comparative static paths.

\footnotetext{
${ }^{4}$ See Judd (1999, p. 179) for an introduction to homotopy methods.
} 
Comparative statics are conceptually simple along regular comparative static paths. One follows the implications of a change in parameters for a single GA structure until at some point a second solution to the min-max problem emerges. According to Theorem 1, this would happen if some the demand set of some buyer expanded or if the price of some good fell to its reservation value. At that point, one would choose the structure that solved the min-max problem along the next segment of the path. The following sections formalize these arguments.

\subsection{Model Types}

We consider a set of models indexed by a parameter vector $\lambda \in \mathbb{R}^{m n+n}$. The first $m n$ components of $\lambda$ are shifters $\xi_{a i}$ of each buyer $x_{a}$ 's utility for each good $y_{i}$. We let $U_{a}\left(y_{i}, p_{i}, \xi_{a i}\right)$ be the utility to $x_{a}$ from the purchase of $y_{i}$ at the price $p_{i}$ when the taste parameter is $\xi_{a i}$. The remaining $n$ components are the reservation prices of the sellers $r_{i}$. We restrict $\lambda$ to lie in a set $\Lambda \subseteq \mathbb{R}^{m n+n}$ in which all the $U_{a}\left(y_{i}, p_{i}, \xi_{a i}\right)$ are strictly monotonic in $\xi_{a i}$. This avoids "flat spots" in which a change in the parameters has no effect on the prices. In addition, we assume that $\Lambda$ is non-empty, open, bounded, and convex..$^{5}$

We make the following smoothness assumption on the utility functions. ${ }^{6}$

Assumption B: The utility functions $U_{a}\left(y_{i}, p_{i}, \xi_{a i}\right)$ are analytic in $p_{i}$ and $\xi_{a i}$ for all $x_{a} \in X$ and $y_{i} \in Y$. Moreover the $U_{a}\left(y_{i}, p_{i}, \xi_{a i}\right)$ are strictly monotonic in $\xi_{a i}$ for all $\lambda \in \Lambda$.

Many of the objects that we have been considering become functions of $\lambda . q(\mu, F, \lambda)$ is the price-induced by $(\mu, F)$ in model $\lambda \in \Lambda . \underline{p}(\lambda)$ is the minimum equilibrium price in model $\lambda \in \Lambda$. $\Phi(\lambda)$ is the GAME set. $\mathcal{M}(\lambda)$ as the edge sets that satisfy condition M.

The next lemma follows from the properties of analytic functions and the Theorem of the Maximum applied to the max-min problem (2.2).

Lemma 2: With Assumptions $\mathrm{A}$ and $\mathrm{B}$, each $q_{i}(\mu, F, \lambda)$ is analytic at $\lambda$ for all $\lambda \in \Lambda$; $\Phi(\lambda)$ is non-empty, compact-valued, and upper-hemicontinuous; and $\underline{p}_{i}(\lambda)$ is continuous for all $y_{i} \in Y$.

\footnotetext{
${ }^{5}$ Taking $\Lambda$ as open avoids the question of how to do comparative statics at the boundary of the parameter space. Boundedness will imply that closed subsets are compact. Convexity implies connectedness, which is a natural assumption when considering continuous paths. Convexity will also prove useful in constructing perturbations of paths.

${ }^{6} \mathrm{~A}$ function $f(x)$ is analytic at a point $x_{0}$, if its Taylor series expansion converges on a neighborhood of $x_{0}$. Almost all commonly used utilty functions are analytic almost everywhere in their domain.
} 
Moreover, it follows from Lojasiewicz's Structure Theorem for Real Varieties (Krantz and Parks [2002], p. 168) that the GAME set is generically single valued. Let $\hat{\Lambda} \equiv\{\lambda \in \Lambda \mid$ $|\Phi(\rho)|>1\}$.

Theorem 2: With Assumptions A and B, $\hat{\Lambda}$ is open and dense in $\Lambda$.

\subsection{Paths}

A typical comparative static exercise involves some well defined change in model parameters from $\lambda_{0}$ to $\lambda_{1}$. We will represent these changes with a path through $\Lambda$ starting from $\lambda_{0}$ and ending at $\lambda_{1}$. Technically, a path is a continuous mapping of the unit interval into parameter space, $\pi:[0,1] \rightarrow \Lambda$, with $\pi(0)=\lambda_{0}$ and $\pi(1)=\lambda_{1}$. Any path induces a correspondence $\phi(z):[0,1] \rightarrow \mathcal{G}$ which maps each $z$ into the GAME set $\phi(z)$.

$$
\phi(z)=\Phi(\pi(z)) .
$$

Note that $\phi$ inherits the properties of $\Phi$. It is upper-hemicontinuous, non-empty and compact valued.

\subsection{Regular Paths}

Even though $\Phi$ is almost everywhere single valued, it will not be possible in general to find a path between two sets of parameters that avoids points at which $\Phi$ takes on multiple values. We consider now what happens at such points.

A simple example shows that in general anything can happen. Suppose that at $\bar{\lambda}$ all buyers have identical preferences and that the path $\pi$ passes through $\bar{\lambda}$, then, in principle, one can transition from any market situation to any other situation with the most minor of changes in model parameters.

This example in which the equilibrium allocation can change in an arbitrary manner relies on a seeming coincidence, with many individuals suddenly become indifferent to various goods the same point along the path. The results of the last section suggest that cases in which there is only one new indifference at a time, or in which one and only one good falls to reservation price at a given point in the parameter space, will be more ordered.

The simplest case is a path at which the number $|\Phi|$ was no higher than 2 all the way along the path. In fact the following cases are the easiest of all.

Definition 4.1. A path $\pi(z)$ is regular if the induced mapping $\phi(z)$ has the following properties: 
1. $\max _{z \in[0,1]}|\phi(z)| \leq 2$.

2. $Z=\{z \in[0, \bar{z}]|| \phi(z) \mid>1\}$ is finite.

Along a regular path, comparative statics is conceptually easy. One travels along a path, working out the implications of a change in parameters for a given GA structure until at some point a new element of $\Phi$ arises. At this point, one chooses to continue with the structure that solves the max-min problem beyond that point. There are a finite number of such switches.

The cardinality results of the last section at least give some hope that there may be many regular paths, given that particular transitions can be shown to ensure cardinality of no more than 2. In the next section, we show in fact that regularity is a generic property of comparative static paths.

\section{The Generic Path is Regular}

\subsection{Analytic Shapes}

Paths can take many forms and the set of possible paths is quite large. In order to keep the analysis manageable, we consider a notion of genericity based on fixing the "shape" of a path and varying the initial condition. We show that given the shape of the path, the initial conditions associated with regular paths form a dense open set. In other words, the generic path is regular.

The shape is a continuous mapping $S:[0,1] \rightarrow \mathbb{R}^{m n+n}$ such that $S(0)=0$. The initial condition is a point in parameter space $\lambda_{0} \in \Lambda$. The pair $\left(\lambda_{0}, S\right)$ define a path $\pi\left(z ; \lambda_{0}, S\right)=$ $\lambda_{0}+S(z)$, which begins at $\lambda_{0}$ and ends at $\lambda_{1}=\lambda_{0}+S(1)$. A path is admissible if $\lambda_{0}+S(z) \in \Lambda$ for all $z \in[0,1]$. Let $\Lambda_{S}$ denote the set of $\lambda_{0}$ for which $\lambda_{0}+S(z)$ is admissible.

For the remainder of the paper we fix the shape $S$ and assume that $\Lambda_{S}$ is a non-empty, open, bounded, and convex subset of $\Lambda$.

Assumption C: Each component of $S(z)$ is analytic on $(0,1)$.

\subsection{Regularity is Generic}

Let $\Lambda_{R}=\left\{\lambda_{0} \in \Lambda_{S} \mid \pi\left(z ; \lambda_{0}, S\right)\right.$ is regular $\}$ denote the set of $\lambda_{0}$ for which the path $\pi\left(z ; \lambda_{0}, S\right)$ is regular. Theorem 3 states the sense in which almost all paths are regular.

Theorem 3: With A-C, $\Lambda_{R}$ is open and dense in $\Lambda_{S}$. 
The proof of Theorem 3 involves a number of steps. The upper hemicontinuity of $\Phi$ can be used to show that the set of non-regular paths is closed. To show that the $\Lambda_{R}$ is dense in $\Lambda_{S}$ we first show that the set of points $\Lambda_{F}=\left\{\lambda_{0} \mid Z=\{z \in[0, \bar{z}]|| \phi(z) \mid>1\}\right.$ is finite $\}$ is dense in $\Lambda_{S}$. To see this, note that each point in $Z$ is associated with an intersection of at least two $q(\mu, F, \pi(z))$. We show that Assumptions $\mathrm{B}$ and $\mathrm{C}$ imply that the $q_{i}(\mu, F, \pi(z))$ are analytic in $z$ for all $z \in(0,1),(\mu, F) \in \mathcal{G}$, and $y_{i} \in Y$. Analytic functions whose intersections have accumulation points must be equal everywhere. We show that whenever $q_{i}(\mu, F, \pi(z))$ are identical for two $(\mu, F)$ we can find a perturbation of $\lambda_{0}$ such that they are different. This establishes $\Lambda_{F}$ is dense in $\Lambda_{S}$. To establish that the set $\Lambda_{R}$ is dense in $\Lambda_{F}$ we show that given $\lambda_{0} \in \Lambda_{F}$, we can perturb $\lambda_{0}$ in such a way that we reduce the number of edges in $\mathcal{E}^{*}$ at points in which $|\phi(z)|>2$.

\section{The Five Market Transitions}

According to Theorem 3, on a regular path there are only a finite number of points at which the GA structure changes and at each of these points of transition $|\phi(z)|=2$. According to Theorem 1, points at which $|\phi(z)|=2$ are associated with an "extra" indifference in the demand graph. The extra indifference could arise either with the expansion of the demand set of a single buyer to a single new good or with a single price falling to $r_{i}$, thereby making superfluous the demand of the buyer who heretofore had supported the good.

The implication is that along the generic path there are only a few ways in which the structure of the market can change. In fact, there are five types of transition. Four are associated with an expansion of a buyer's demand set. They differ in the position of the good demanded, whether it is (1) unallocated, (2) allocated and a root good in another component, (3) allocated and a predecessor in the same component, or (4) none of the above. The last case is associated with a contraction in the graph satisfying the conditions of the Corollary 1 . This happens when the price of a good falls to its reservation level. Below we discuss each case in terms of the impact on the GA-structure, since it is this that illustrates most clearly how, if at all, the allocation changes, and how the structure of market interdependence changes at critical transition points.

We present the cases in the order of complexity. In each case, we describe what happens at a point of market transition $z$. There is some GA structure $(\mu, F)$ which has characterized the market prior to $z$. At $z$ either some buyer $\bar{x}$ becomes indifferent to some good $\bar{y}$ or the price of some good $\bar{y}$ falls to $\bar{r}$. We describe the relationship between $(\mu, F)$ and $\left(\mu^{\prime}, F^{\prime}\right)$, the GA structure that characterizes the market after $z$. It is useful to let $\bar{\mu}$ denote the good assigned $\bar{x}$ 
by $\mu$.

\subsection{Graft}

Grafting occurs when $\bar{x}$ 's demand correspondence expands to include a good $\bar{y}$ that is the root of another tree. The tree with $\bar{y}$ as its root is incorporated in $\bar{x}$ to create a single larger tree.

An example of grafting is illustrated in Figure 5. Figure 5(a) shows the initial GA structure $(\mu, F)$. There are five goods. The only goods that are labeled are $\bar{y}$, the root of the second tree, and $\bar{\mu}$, the good in the first tree whose buyer becomes indifferent to $\bar{y}$. This indifference is illustrated by the light directed edge from $\bar{\mu}$ to $\bar{y}$. It is clear that $\bar{y}$ can be priced in two ways: it can be set at reservation or it can be priced using $\bar{x}$ 's indifference. This latter GA structure is illustrated in Figure 5(b). It involves solidifying the link from $\bar{\mu}$ to $\bar{y}$ and the uprooting of $\bar{y}$, as illustrated by the circular as opposed to square node.

In general, grafting is characterized by the following rules. First, there is no change in the equilibrium allocation, $\mu^{\prime}=\mu$. Second, $F^{\prime}$ differs from $F$ in just two ways: the added edge from $\bar{\mu}$ to $\bar{y}, E\left(F^{\prime}\right)=E(F) \cup(\bar{\mu}, \bar{y})$, and the removal of $\bar{y}$ from the root set, $R\left(F^{\prime}\right)=R(F) \backslash \bar{y}$.

\subsection{Prune and Plant}

Pruning is the opposite of grafting. It involves the division of one tree into two separate trees. It occurs when some good $\bar{y}$ that used to be above reservation price falls to this price, and at that point becomes a root good. This occurrence is illustrated in Figure 6(a). The new GA structure involves severing of the proximate link to the new root good, as illustrated in Figure 6(b). Again, there is no change in the allocation.

\subsection{Prune and Graft}

As its name indicates, prune and graft combines both pruning and grafting. This case involves separating a branch from a tree and attaching it somewhere else. This is the "none of the above" case described above, in which $\bar{y}$ is allocated, but not a root good or a predecessor to $\bar{\mu}$. As in the cases of pruning and grafting there is no change in the allocation. As with pruning, the link between $\bar{y}$ and its direct predecessor in $F$ is cut. As with grafting, $\bar{y}$ is grafted onto the new directed edge from $\bar{\mu}$ to $\bar{y}$.

Two different cases with this common structure are illustrated in figures 7 (a) and 7(b), with the difference being that in the first case $\bar{\mu}$ and $\bar{y}$ are in different trees, which in the second case they are in the same tree, with $\bar{\mu}$ being a predecessor of $\bar{y}$. It is clear in each case, the addition 
of the edge $(\bar{\mu}, \bar{y})$ creates two and only two ways to price good $\bar{y}$, one corresponds to the initial graph $F$, the other to $F^{\prime}$.

Prune and Graft is characterized by the following rules: $\mu^{\prime}=\mu ; R\left(F^{\prime}\right)=R(F)$; and $E\left(F^{\prime}\right)=$ $\left\{E(F) \backslash\left(y^{\prime}, \bar{y}\right)\right\} \cup(\bar{\mu}, \bar{y})$ where $y^{\prime}$ is the direct predecessor of $\bar{y}$ in $F$.

\subsection{Cyclic Reversal}

The two final cases both involve changes both in the allocation of goods, as well as the nature of the interdependence. Cyclic Reversal occurs when both $\bar{\mu}$ and $\bar{y}$ are elements of the same tree, and $\bar{y}$ is a predecessor of $\bar{\mu}$.

A simple example with three goods and three buyers is illustrated in Figure 8(a). Initially, each buyer is matched with the correspondingly numbered good: $\mu_{i}=y_{i}$, and the GA structure involves two directed edges: $E(F)=\left\{\left(y_{1}, y_{2}\right),\left(y_{2}, y_{3}\right)\right\}$. In this example, $\bar{\mu}=y_{3}$ and $\bar{y}=y_{1}$, as illustrated by the corresponding light edge. Note that the addition of the directed edge, $\left(y_{3}, y_{1}\right)$ creates a cycle in which each buyer is indifferent between his assigned good and its direct successor. A cyclic permutation of the allocation therefore keeps each buyer in his demand set. It is clear that we can generate the same price vector as follows. First, allocate $\bar{x}$ to $y_{3}$ and use his indifference to price $y_{3}$. Next take the buyer allocated to $y_{2}$, shift him to $y_{3}$ and use his indifference to price $y_{2}$. Finally, take the buyer allocated to $y_{1}$, shift him to $y_{2}$. This GA structure is illustrated in figure $8(\mathrm{~b})$.

Formally, cyclic reversal involves the following steps. Add the edge $(\bar{\mu}, \bar{y})$ to $E(F)$. This creates a directed cycle $C=\left\{y_{1}, y_{2}, \ldots, y_{k}, y_{k+1}\right\}$ with $y_{1}=y_{k+1}=\bar{y}, y_{k}=\bar{\mu}$, and $\left(y_{i}, y_{i+1}\right) \in$ $E(F) \cup(\bar{\mu}, \bar{y})$ for $i \in\{1, \ldots k-1\}$. The new allocation $\mu$ involves rotating all buyers allocated to a good in $C$ to its direct successor

$$
\mu_{a}^{\prime}=\left\{\begin{array}{cc}
\mu_{a} & \text { if } \mu_{a} \notin C \\
y_{i+1} & \text { if } \mu_{a}=y_{i} \in C
\end{array}\right.
$$

The new graph $F^{\prime}$ is derived from the old graph $F$ in a series of steps by first reversing all of the edges in the cycle $C$ by replacing $\left(y_{i}, y_{i+1}\right)$ for all $y_{i}, y_{i+1} \in C$ with $\left(y_{i+1}, y_{i}\right)$, then eliminating $\left(y_{2}, y_{1}\right)$ and finally, replacing all $\left(y_{i}, y_{j}\right)$ with $y_{i} \in C$ and $y_{j} \notin C$ with $\left(y_{i+1}, y_{j}\right)$ so that all goods not in $P$ are priced by the same buyers in $\left(\mu^{\prime}, F^{\prime}\right)$ as in $(\mu, F)$. 


\subsection{Shift and Replant}

Shift and Replant occurs when $\bar{y}$ is unallocated. It is similar to cyclic reversal except that instead of relocating buyers around a cycle, we relocate them along a path.

Figure 9 (a) provides an example. There are four goods. The first three are allocated to like-numbered buyers. The forth good $\bar{y}$ is unallocated and hence a root good as indicated by the rectangle to which no one is assigned. The initial GA structure $(\mu, F)$ involves directed edges $\left(y_{1}, y_{2}\right)$ and $\left(y_{2}, y_{3}\right)$. It is buyer $x_{3}=\bar{x}$ who then becomes indifferent with the unoccupied good $\bar{y}$. Note that we can support the same price vector, by first shifting the buyers along the connected path between the two root goods, with $\bar{x}$ to $\bar{y}, x_{2}$ to $y_{3}$ and $x_{1}$ to $y_{2}$, and then using $\bar{x}$ 's indifference to price $y_{3}, x_{2}$ 's to price $y_{2}$. $y_{1}$ is unallocated and hence priced at reservation. This situation is illustrated in Figure 9(b).

Formally, shift and replant is characterized by the following operations. We first add the edge $(\bar{\mu}, \bar{y})$ and let $P=\left\{y_{1}, y_{2}, \ldots, \bar{\mu}, \bar{y}\right\}$ denote the path in $E(F) \cup(\bar{\mu}, \bar{y})$ beginning at $y_{1} \in R(F)$ and ending with $\bar{y}$. The change in the allocation $\mu$ involves shifting all buyers allocated to a good in $P$ to its direct successor:

$$
\mu_{a}^{\prime}=\left\{\begin{array}{cc}
\mu_{a} & \text { if } \mu_{a} \notin P \\
y_{i+1} & \text { if } \mu_{a}=y_{i} \in P
\end{array}\right.
$$

The new graph $F^{\prime}$ is derived from the old graph $F$. First, reverse all of the edges in the path $P$ by replacing $\left(y_{i}, y_{i+1}\right)$ for all $y_{i}, y_{i+1} \in P$ with $\left(y_{i+1}, y_{i}\right)$. Then eliminate $\left(y_{2}, y_{1}\right)$. Finally, replace all $\left(y_{i}, y_{j}\right)$ with $y_{i} \in P$ and $y_{j} \notin P$ with $\left(y_{i+1}, y_{j}\right)$, so that all goods not in $P$ are priced by the same buyers in $\left(\mu^{\prime}, F^{\prime}\right)$ as in $(\mu, F)$.

\subsection{The Fundamental Theorem of Calculus for Allocation Markets}

The above results allow us to express discrete changes in prices as the integral of infinitesimal market changes. Consider a change for $\lambda_{0}$ to $\lambda_{1}$ along a regular path $\pi(z)$. Let $\hat{Z}=\{z \in$ $[0,1] \mid \phi(z-\varepsilon) \neq \phi(z+\varepsilon)\}$ denote the set of potential switch points, and label the elements of $\hat{Z}=\left\{z_{1}, \ldots z_{S}\right\}$. Let $\left(\mu_{s}, F_{s}\right)$ denote the unique element of $\phi(z)$ over the interval $\left(z_{s}, z_{s+1}\right)$, where it is understood that $z_{0}=0$ and $z_{S+1}=1$. Prior results imply that the change in the price of good $y_{i}$ may be obtained by integrating the change in price over each subinterval and 
summing over subintervals:

$$
p_{i}\left(\lambda_{1}\right)-p_{i}\left(\lambda_{0}\right)=\sum_{s=0}^{S} \int_{z_{s}}^{z_{s+1}}\left(\left.\frac{d p_{i}}{d \lambda(z)}\right|_{\left(\mu_{s}, F_{s}\right)} \frac{d \lambda(z)}{d z}\right) d z
$$

Here the derivative $d p_{i} / d \lambda(z)$ is taken with respect to the current GA structure $\left(\mu_{s}, F_{s}\right)$.

\section{An Algorithm for Computing Competitive Equilibria}

The maximization in problem (2.2) is over a very large set and may be difficult to perform in practice. A generalization of Cayley's theorem states that for each allocation $\mu$ there are

$$
\sum_{k=1}^{m}\left(\begin{array}{c}
m \\
k
\end{array}\right) k m^{m-1-k}
$$

different forests of rooted trees on the $m$ allocated goods. ${ }^{7}$ On top of this there are $n ! /(n-m !)$ ways to allocate buyers to goods. If $n=m=10$, we get more than $8.5 \times 10^{15}$ different GAstructures. If $n=m=1000$, we get more than $9 \times 10^{3609}$.

We have shown how to use homotopy methods to move between any two competitive equilibria. This insight may be used to compute equilibria and avoid searching through such a large set. We only need a simple equilibrium to start from. One convenient initial equilibrium is the null equilibrium in which the preference parameters are such that each buyer prefers a different good at reservation prices.

The algorithm works in the following way. Given any set of parameters, we initialize this algorithm by raising the parameter $\xi_{a, a}$ for each buyer $x_{a}$ high enough that each buyer $x_{a}$ prefers $y_{a}$ to all other goods when prices are set at reservation levels. At this level of reservation utility, the minimum price equilibrium has all prices at reservation level and all buyers allocated to their preferred goods. There is a unique GA-structure corresponding to this equilibrium. It involves the equilibrium allocation and a null graph in which all goods are root goods and there are no edges. This equilibrium is our starting point. We can then lower the $\xi_{a, a}$ to their original levels, tracking GA-structures that correspond to the minimum price equilibrium. Note that lowering the $\xi_{a, a}$ has the effect of raising prices monotonically to their final level.

This algorithm is related to the ascending auction mechanism of Demange, Gale and Sotomayor (1986). They consider minimum price equilibria in a model with transferable utility

\footnotetext{
${ }^{7}$ See Aigner and Ziegler [2003, 3rd edition, p. 178].
} 
and discrete prices. Their algorithm involves increasing the prices of all goods in minimal overdemanded sets by one unit until supply and demand are brought into balance. The key complication that non-transferable utility introduces is that the same price change affects the demands of different buyers differently. The challenge is to find a way of raising prices that does not completely alter the balance between supply and demand, while at the same time keeping track of the resulting changes in the allocation. The GA structures provide such a mechanism.

There are several attractive features of this algorithm. First, it terminates by precisely identifying the minimum equilibrium price. This is not the case with approximation methods that are often employed in computing economic equilibria (e.g. Scarf (1973)). ${ }^{8}$ Second, the algorithm is likely to be relatively fast in many practical applications. There is a sense in which the algorithm is minimal: it searches only through the set of potential solutions for some set of utility parameters and by-passes the mass of entirely unsuitable price vectors. This mirrors the situation with the simplex method, in which one searches only through the set of extreme points of the feasible set, all of which are optimal for some vector of resources.

\subsection{From Continuous to Discrete}

Practical implementation of the algorithm requires discretizing $z$. Figure 10 illustrates one possibility. We begin with a known solution to to the model $\left(\mu_{1}, F_{1}\right)$ at $z_{1}=0$. At each step in the algorithm our current candidate solution $\left(\mu_{1}, F_{1}\right)$ which is known to generate the minimum equilibrium price at some point $z_{1},\left(\mu_{1}, F_{1}\right) \in \Phi\left(z_{1}\right)$, and test whether it generates the minimum equilibrium price at some further point $z_{2}$. Initially, we take $z_{2}=1$.

If $\left(\mu_{1}, F_{1}\right) \in \Phi\left(z_{2}\right)$, this test succeeds and we reset $z_{1}=z_{2}$ and $z_{2}=1$. If $z_{1}=1$, we are finished. Otherwise, we test $\left(\mu_{1}, F_{1}\right)$ at $z_{2}=1$.

We will say that there is a "violation of competitive equilibrium" at $z_{2}$ if, given $q\left(\mu_{1}, F_{1}, \pi\left(z_{2}\right)\right)$, a buyer prefers a good to the one that he is allocated or if there is good whose price falls below reservation. The test succeeds if there is no violations. If there is a single violation of competitive equilibrium when applying $\left(\mu_{1}, F_{1}\right)$ at $z_{2}$, meaning a single buyer who prefers a single good or a single good whose price falls below reservation, then we construct $\left(\mu_{2}, F_{2}\right)$, which is the alternative GA structure suggested by treating the violation as an indifference and making the appropriate market transition as in the last section, and test $\left(\mu_{2}, F_{2}\right)$ at $z_{2}$. If $\left(\mu_{2}, F_{2}\right) \in \Phi\left(z_{2}\right)$, we update $\left(\mu_{1}, F_{1}\right)=\left(\mu_{2}, F_{2}\right)$, as well as $z_{1}=z_{2}$, and $z_{2}=1$ and proceed as before. If there are multiple violations of competitive equilibrium at $q\left(\mu_{1}, F_{1} ; \lambda\left(z_{2}\right)\right)$ or if $\left(\mu_{2}, F_{2}\right) \notin \Phi\left(z_{2}\right)$, then

\footnotetext{
${ }^{8}$ Miyake (2003) also provides an algorithm with this property.
} 
we stepped over multiple market transitions. We then try taking a smaller step: we update $z_{2}=\left(z_{1}+z_{2}\right) / 2$ and try $\left(\mu_{1}, F_{1}\right)$ again.

Given Theorem 3, which states that there are a finite number of points of transition on a generic path, this algorithm converges. Eventually, the step size is reduced to the point that $\left(z_{1}, z_{2}\right)$ contains a single market transition and $\left(\mu_{2}, F_{2}\right) \in \Phi\left(z_{2}\right)$

\section{Conclusion}

In this paper and it companion, we have introduced a new mathematical apparatus for understanding allocation markets with nontransferable utility. We are currently extending the work to a dynamic context and solving for the reallocation of objects over time. Our methods may also apply in other areas. 


\section{References}

[1] Caplin, Andrew, and John Leahy (2010), "A Graph Theoretic Approach to Allocation Markets," New York University working paper.

[2] Demange, Gabrielle, and David Gale (1985), "The Strategy Structure of Two-Sided Matching Markets," Econometrica 53, 873-888.

[3] Demange, Gabrielle, and David Gale, and Marilda Sotomayor (1986), "Multi-Item Auctions," The Journal of Political Economy 94, 863-873.

[4] Judd, Kenneth, (1999) Numerical Methods in Economics, Cambridge: MIT Press.

[5] Kaneko, Mamoru, (1982), "The Central Assignment Game and the Assignment Markets, Journal of Mathematical Economics 10, 205-232.

[6] Krantz, Steven, and Harold Parks (2002), "A Primer of Real Analytic Functions," Boston: Birhauser.

[7] Kuhn, Harold (1955), "The Hungarian Method for the Assignment Problem," Naval Research Logistics Quarterly 2, 83-97.

[8] Miyake, Mitsunobu (2003), "Precise Computation of a Competitive Equilibrium of the Discrete Land Market Model," Regional Science and Urban Economics 33, 721-743.

[9] Munkres, James (1957), "Algorithms for the Assignment and Transportation Problems," Journal of the Society of Industrial and Applied Mathematics 5, 32-38.

[10] Scarf, Herbert (1973), The Computation of Economic Equilibria, New Have: Yale University Press.

\section{Appendix: Proofs}

Lemma 1: There exists a bijection $\eta: \mathcal{M} \rightarrow \Phi$ so that

$$
|\Phi|=|\mathcal{M}|
$$

Proof: We identify a mapping $\eta: \mathcal{M} \rightarrow \Phi$ and show it to be a bijection. Given $\mathcal{E} \in \mathcal{M}$, we identify $\eta(\mathcal{E})=(\mu(\mathcal{E}), F(\mathcal{E}))$ starting with the allocation. We first match all goods of degree one. 
If there are goods remaining in any set $Y_{l}^{\mathcal{E}}$ after the removal of these goods and their matched buyers, there is as least one of degree one, since at least one edge to the unmatched goods is removed when the prior match is created, due to the connectedness of the components. This process ends by uniquely specifying $\mu(\mathcal{E}) \in M$ in no more than $\max \left|Y_{l}^{\mathcal{E}}\right|$ steps. We construct the graph $F(\mathcal{E})=(Y, R, E)$ by first setting $R$ equal to the union of $Y_{0}^{\mathcal{E}}$ and the valence 1 good in each set $Y_{l}^{\mathcal{E}}$ for $l \geq 1$. We then define $E=\left\{\left(y_{i}, y_{k}\right) \mid\left(x_{a}, y_{i}\right),\left(x_{a}, y_{k}\right) \in \mathcal{E}\right.$ and $\left.\mu_{a}=y_{i}\right\}$. Since each non-root good is allocated and has degree two, it has a unique predecessor in $E$. Since each is connected to the root good by construction, each component is a tree. To establish that $\eta: \mathcal{M} \rightarrow \Phi$, note by construction $q(\mu(\mathcal{E}), F(\mathcal{E}))=\underline{p}$, that $(\mu(\mathcal{E}), F(\mathcal{E}))$ identifies a minimum price equilibrium, and that all goods to which a directed edge points are allocated.

It remains to establish that $\eta: \mathcal{M} \rightarrow \Phi$ is onto $\Phi$. Consider any $(\tilde{\mu}, \tilde{F}) \in \Phi$. We identify the element $\gamma(\tilde{\mu}, \tilde{F})=\eta^{-1}(\tilde{\mu}, \tilde{F}) \in \mathcal{M}$ such that $\eta(\tilde{\mathcal{E}})=(\tilde{\mu}, \tilde{F})$ in stages. First, we generate a bipartite graph on $X \cup Y$ with bipartition $(X, Y)$ by joining each buyer with the good to which they are matched, placing $\left(x_{a}, \tilde{\mu}_{a}\right) \in \tilde{\mathcal{E}}$, and then placing $\left(x_{a}, y_{b}\right) \in \tilde{\mathcal{E}}$ if $\left(\tilde{\mu}_{a}, y_{b}\right) \in F$. To see that $\gamma(\tilde{\mu}, \tilde{F}) \in \mathcal{M}$, note first that $q(\tilde{\mu}, \tilde{F})=\underline{p}$, hence this graph is a subset of $\mathcal{E}^{*}$. To see that it satisfies the remaining aspects of condition $M$, look at each tree in the forest and note that there every node of the tree has one and only one buyer due to the insistence that downstream as well as upstream goods are allocated. Each such tree in the forest thereby is mapped to a connected set $\left(X_{l}^{\mathcal{E}}, Y_{l}^{\mathcal{E}}\right)$ for $1 \leq l \leq L$ that is disconnected from all others, and in which the equal cardinality condition M2 is satisfied. The isolated set $Y_{0}^{\mathcal{E}}$ comprises all goods that are not in demand by anyone. By construction, there is one and only one good of degree 1 in each partition set $Y_{l}^{\mathcal{E}}$ for $l \geq 1$, and all others have degree 2 . Moreover, by construction all goods of valence 1 are root goods for which $q_{j}^{*}=r_{j}$. By construction, goods of degree 0 also satisfy $q_{j}^{*}=r_{j}$. Hence $\underline{p}_{i}>r_{i}$ implies that the degree of $y_{i}$ is 2 . This confirms that $\gamma(\tilde{\mu}, \tilde{F}) \in \mathcal{M}$. Finally, it is direct from the construction that $\eta(\tilde{\mathcal{E}})=(\tilde{\mu}, \tilde{F})$, establishing that indeed $\gamma: \Phi \rightarrow \mathcal{M}$ is the inverse function of $\eta: \mathcal{M} \rightarrow \Phi$.

Corollary 1: $\mathcal{E}^{*} \in \mathcal{M}$ and $d^{*}\left(y_{i}\right)=2 \Longrightarrow \underline{p}_{i}>r_{i}$ then $|\Phi|=1$.

Proof: Suppose that $\mathcal{E}^{*} \in \mathcal{M}$ and all $y_{i}$ with $d^{*}\left(y_{i}\right)=2$ have $\underline{p}_{i}>r_{i}$. We show that $\mathcal{M}$ has one element. Lemma 1 then implies $|\Phi|=1$. That there is no second element follows from the observation that since $\mathcal{E}^{*} \in \mathcal{M}$ all goods have degree less than or equal to two. Removing any edge from the demand graph $D$ reduces the degree of some good. If the degree falls from one to zero, then the number of goods with degree greater than zero is less than the number of buyers 
in contradiction of condition M4. If a good has degree two in $D$, then by assumption its price is above reservation. Reducing the degree of such a good contradicts condition M5.

Theorem 1: $|\Phi|=2$ if and only if there exists $(\bar{x}, \bar{y}) \in \mathcal{E}^{*}$ such that $\mathcal{E}^{*} /(\bar{x}, \bar{y}) \in \mathcal{U}$.

Proof: (If) Suppose that there exists $(\bar{x}, \bar{y}) \in \mathcal{E}^{*}$ such that $\mathcal{E}^{*} /(\bar{x}, \bar{y}) \in \mathcal{U}$. To establish $|\Phi|=2$, let $\mathcal{E}_{1}=\mathcal{E}^{*} /(\bar{x}, \bar{y})$. We consider a number of sub-cases and in each such case identify $\mathcal{E}_{2} \neq \mathcal{E}_{1}$ with $\mathcal{E}_{2} \in \mathcal{M}$. We then show that there is no third member $\mathcal{E}_{3} \in \mathcal{M}$. The argument depends on features of $\bar{\mu} \in Y$, the good matched to $\bar{x}$ in the unique match associated with $\mathcal{E}_{1} \in \mathcal{U}$.

1. If $\bar{y} \in Y_{0}^{\mathcal{E}_{1}}$, let $\bar{\sigma} \in Y$ be the root good in the set $Y_{l}^{\mathcal{E}_{1}}$ such that $\bar{x} \in X_{l}^{\mathcal{E}_{1}}$, and let $\mu^{-1}(\bar{\sigma}) \in X_{l}^{\mathcal{E}_{1}}$ be the buyer matched to that good. Define $\mathcal{E}_{2}=\mathcal{E}^{*} /\left\{\mu^{-1}(\bar{\sigma}), \bar{\sigma}\right\}$. Note that $\mathcal{E}_{2} \in \mathcal{U}$ by construction, since the only change is the replacement of $\bar{\sigma} \in Y_{l}^{\mathcal{E}_{1}}$ with the good $\bar{y} \in Y_{l}^{\mathcal{E}_{2}}$, also of valence 1 . Now suppose that there exists some third element $\mathcal{E}_{3} \in \mathcal{M}$, with $\mathcal{E}_{3} \notin\left\{\mathcal{E}_{1}, \mathcal{E}_{2}\right\}$. Note that this set must contain both $\left\{\mu^{-1}(\bar{\sigma}), \bar{\sigma}\right\}$ and $(\bar{x}, \bar{y})$ given that uniqueness holds with either element excluded. Hence some other edge from those joining $X_{l}^{\mathcal{E}_{1}}$ and $Y_{l}^{\mathcal{E}_{1}}$ must be removed, thereby reducing the degree of some element other than $\bar{\sigma}$ or $\bar{y}$ either to 1 or to zero. This contradicts the fact that this good must have $\underline{p}_{i}>r_{i}$, which implies that its degree in $\mathcal{E}_{2}$ must be 2 by M4.

2. Suppose $(\bar{\mu}, \bar{y}) \subset Y_{l}^{\mathcal{E}_{1}}$ for some $l \geq 1$. Note in this case that $\mathcal{E}^{*}$ itself has a cycle, since it $X_{l}^{\mathcal{E}_{1}} \cup Y_{l}^{\mathcal{E}_{1}}$ has as many edges as vertices in $\mathcal{E}^{*}$ : this cycle is unique, since all cycles must contain $\{\bar{x}, \bar{y}\}$, so that if there were two of them, then there would have been one prior to the addition of $\{\bar{x}, \bar{y}\}$ (geometrically clear). Now define $\left\{x^{C}, \bar{y}\right\}$ as the unique edge other than $(\bar{x}, \bar{y})$ heading to $\bar{y}$ in this unique cycle in the graph $\left(X \cup Y, \mathcal{E}^{*}\right)$. Define $\mathcal{E}_{2}=\mathcal{E}^{*} /\left\{x^{C}, \bar{y}\right\}$. By construction, $\mathcal{E}_{2} \neq \mathcal{E}_{1}$ satisfies $\mathcal{E}_{2} \in \mathcal{U}$, since all goods have the same valence in $\mathcal{E}_{2}$ and $\mathcal{E}_{1}$, and the associated partitions are identical. Now suppose that there exists some third element $\mathcal{E}_{3} \in \mathcal{M}$, with $\mathcal{E}_{3} \notin\left\{\mathcal{E}_{1}, \mathcal{E}_{2}\right\}$. This set must contain both $\left\{x^{C}, \bar{y}\right\}$ and $(\bar{x}, \bar{y})$ given that uniqueness holds with either element excluded. Hence some other edge in the unique cycle must be removed. In so doing, one reduces the degree of some good other than $\bar{y} \in Y_{l}^{\mathcal{E}_{1}}$ below 2 . This again contradicts the fact that this good must have $p_{i}>r_{i}$, which implies that its degree in $\mathcal{E}_{2}$ must be 2 by M4.

3. Suppose $\bar{\mu} \in Y_{l}^{\mathcal{E}_{1}}$ and $\bar{y} \in Y_{m}^{\mathcal{E}_{1}}$ with $m \notin\{0, l\}$. There are two sub-cases depending on the valence of $\bar{y}$. 
1. If $\bar{y}$ is of valence 2 in $\mathcal{E}_{1}$ then define $\mathcal{E}_{2}=\mathcal{E}^{*} /\left\{x^{N}, \bar{y}\right\}$, where $x^{N}$ is the element in $Y_{l}^{\mathcal{E}}$ that is not matched to $\bar{y}$ in unique match $\bar{\mu}\left(\mathcal{E}_{1}\right)$. Note that $\mathcal{E}_{2} \in \mathcal{U}$, since one now has two sets with root goods of valence 1 at reservation and all others of valence 2 , in line with the uniqueness condition. Again any third element $\mathcal{E}_{3} \in \mathcal{M}$, with $\mathcal{E}_{3} \notin\left\{\mathcal{E}_{1}, \mathcal{E}_{2}\right\}$ must include both $\left\{x^{N}, \bar{y}\right\}$ and $(\bar{x}, \bar{y})$, but exclude some other element of the edge set corresponding to $\left(X_{l}^{\mathcal{E}} \cup X_{m}^{\mathcal{E}}, Y_{l}^{\mathcal{E}} \cup Y_{m}^{\mathcal{E}}\right)$. However this happens, it calls for there to be at least two elements of valence 1 since the resulting set is disconnected, but there is by assumption only one good at reservation price in the set, a contradiction.

2. If $\bar{y}$ is of valence 1 in $\mathcal{E}$, then $\mathcal{E}^{*} \in \mathcal{M}$ since the joint set $Y_{l}^{\mathcal{E}} \cup Y_{m}^{\mathcal{E}}$ has all but one good of valence 1 , which good is at reservation price. Now suppose that there exists some third element $\mathcal{E}_{3} \in \mathcal{M}$, with $\mathcal{E}_{3} \notin\left\{\mathcal{E}_{1}, \mathcal{E}^{*}\right\}$. Since $\mathcal{E}^{*} /(\bar{x}, \bar{y})$ satisfies the uniqueness condition, this set must contain $(\bar{x}, \bar{y})$ and exclude something from the edge set corresponding to $\left(X_{l}^{\mathcal{E}} \cup X_{m}^{\mathcal{E}}, Y_{l}^{\mathcal{E}} \cup Y_{m}^{\mathcal{E}}\right)$. As before, it will create some good with $\underline{p}_{i}>r_{i}$ yet with degree strictly, a contradiction.

4. An adaptation of case $3(\mathrm{~b})$ works in case 2 , with $\mathcal{E}^{*} \in \mathcal{M}$ and existence of one and only one $y_{j} \in Y$ such that $d^{*}\left(y_{j}\right)=2$ and $\underline{p}_{j}=r_{j}$. Since $\mathcal{E}^{*} \in \mathcal{M}$, there is a unique allocation $\mu\left(\mathcal{E}^{*}\right)$ consistent with $\mathcal{E}^{*}$. Identify the unique element $x_{j}^{N} \in X$ that is to linked $y_{j}$ but not matched to it according to this allocation. Note that $\mathcal{E}^{*} /\left\{x_{j}^{N}, y_{j}\right\}=\mathcal{E}_{1} \in \mathcal{U}$, since $y_{j}$ has valence 1 and is a root good with the removal of its second edge, with all of the matches preserved. Now suppose that there exists some third element $\mathcal{E}_{3} \in \mathcal{M}$, with $\mathcal{E}_{3} \notin\left\{\mathcal{E}_{1}, \mathcal{E}^{*}\right\}$. This set must contain $\left\{x_{j}^{N}, y_{j}\right\}$ given that uniqueness holds with it excluded, and it cannot be the whole set, since this is $\mathcal{E}^{*}$. Hence some other edge must be removed from the partition element $X_{l}^{\mathcal{E}^{*}} \cup Y_{l}^{\mathcal{E}^{*}}$ that contains $y_{j}$. If this is of degree 1 , then there is one too few good: if it is any of the degree 2 goods, then one reduces the degree of some good with $\underline{p}_{i}>r_{i}$ below 2 , a contradiction.

(Only If) If $|\Phi|=2$ then certainly $\mathcal{E}^{*}$ has some subset that satisfies the uniqueness condition of corollary 1 . It can have at most one more edge, since if it had two, then we know from above proof how to create at least three members of $\mathcal{M}$. If it has no more edges than $\mathcal{E}^{*}$, then it must contain at least one more good at reservation price than in that minimum, and we know that it cannot contain more than that, since then the proof above shows can find at least one for each additional good at reservation. This completes the proof. $\square$ 
Lemma 2: With Assumptions A and B, each $q_{i}(\mu, F, \lambda)$ is analytic in $\lambda \in \Lambda ; \Phi(\lambda)$ is non-empty, compact-valued, and upper-hemicontinuous; and each $\underline{p}_{i}(\lambda)$ is continuous.

Proof: We prove the $q_{i}(\mu, F, \lambda(z))$ are analytic by induction. Consider first $y_{i} \in R(F) \equiv A_{0}$. $g_{i}=r_{i}$ which is trivially analytic in $\lambda$. Now suppose that for all $y_{i} \in A_{n}, g_{i}$ are analytic functions of $\lambda$. Consider $S$, the set of direct successors to $A_{n}$. $S=\left\{y_{i} \notin A_{n} \mid\left(y_{j}, y_{i}\right) \in E(F)\right.$ for some $\left.y_{j} \in A_{n}\right\}$. Let $\mu_{a}=y_{j}$. Now $q_{j}$ is defined implicitly by the indifference of $x_{a}$ :

$$
U_{a}\left(y_{i}, q_{i}, \xi_{a i}\right)=U_{a}\left(y_{j}, q_{j}, \xi_{a j}\right)
$$

where according to Assumption B, $U_{a}\left(y_{j}, q_{j}, \xi_{a i}\right)$ and $U_{a}\left(y_{i}, q_{i}, \xi_{a j}\right)$ are analytic functions of their second and third arguments and strictly monotonic in the third. Assumption A ensures that $U_{a}$ is strictly monotonic in the second. It follows from the Real Analytic Implicit Function Theorem (Krantz and Parks, 2002, p. 35) that $q_{i}$ is an analytic function of $\lambda$. This completes the induction step.

That $\Phi(\lambda)$ is non-empty, compact-valued, and upper-hemicontinuous; and each $\underline{p}_{i}(\lambda)$ is continuous follows from Theorem 3 in CL.

The following Lemmas are used in the proof of Theorem 3. The proof of Theorem 3 is divided between three propositions which together establish that $\Lambda_{R}$ is open and dense in $\Lambda_{S}$.

Lemma 3: For each $y_{i}, q_{i}(\mu, F, \lambda(z))$ is real analytic on $z \in(0,1)$.

Proof: According to Lemma 2, Assumptions A and B imply that $q_{i}(\mu, F, \lambda)$ is real analytic on $\lambda \in \Lambda$. Assumption $\mathrm{C}$ states that $\lambda$ is real analytic on $z \in(0,1)$. That the $q_{i}(\mu, F, \lambda(z))$ are analytic follows from the observation that compositions of analytic functions are analytic (Kranz and Parks, p.19). $\square$

Lemma 4: Given any three GA structures, $\left(\mu_{1}, F_{1}\right),\left(\mu_{2}, F_{2}\right)$, and $\left(\mu_{3}, F_{3}\right)$, let $\tilde{\Lambda}$ denote the set $\lambda_{0}$ such that $\left|\phi\left(z ; \lambda_{0}, S\right) \cap\left\{\left(\mu_{1}, F_{1}\right),\left(\mu_{2}, F_{2}\right),\left(\mu_{3}, F_{3}\right)\right\}\right| \leq 2$ for all $z$. $\tilde{\Lambda}$ is open.

Proof: Consider $\bar{\lambda}_{0} \in \tilde{\Lambda}$ and let $\Lambda^{B}=\Lambda\left\{\lambda \in \Lambda \mid\left\{\left(\mu_{1}, F_{1}\right),\left(\mu_{2}, F_{2}\right),\left(\mu_{3}, F_{3}\right)\right\} \in \Phi(\lambda)\right\}$. The upper hemicontinuity of $\Phi$ implies then implies $\Lambda^{B}$ is closed. Since $\Lambda^{B}$ is bounded, it is compact.

Given $\lambda, \lambda^{\prime} \in \Lambda$, let $d\left(\lambda, \lambda^{\prime}\right)$ denote the Euclidian distance between $\lambda$ and $\lambda^{\prime}$ and let $d(z)=$ $\min _{\lambda \in \Lambda^{B}} d\left(\pi\left(z ; \bar{\lambda}_{0}, S\right), \lambda\right) . \Lambda^{B}$ is compact so the minimum problem is well defined. $d\left(\lambda, \lambda^{\prime}\right)$ is continuous in both arguments, so the Theorem of the Maximum states that $d(z)$ is continuous. 
Since $z \in[0,1], d(z)$ has a minimal value. Let $\delta=\min d(z)$. Since the path $\pi$ and the set $\Lambda^{B}$ are disjoint $\delta>0$. It follows that a ball around $\bar{\lambda}_{0}$ with radius $\delta / 2$ is contained in $\tilde{\Lambda}$. This establishes that $\tilde{\Lambda}$ is open.

Lemma 5: Given $\lambda \in \Lambda$ such that $|\Phi(\lambda)|>2$, there exists $\left\{\left(\mu^{1}, F^{1}\right),\left(\mu^{2}, F^{2}\right)\left(\mu^{3}, F^{3}\right)\right\} \subseteq \Phi(\lambda)$ and $l \in \mathbb{R}^{m n+n}$ with $d(l, 0)=1$ such that given any $\chi>0$ and $\delta=\chi l$ such that $\lambda+\delta \in \Lambda$, $\left\{\left(\mu^{1}, F^{1}\right),\left(\mu^{2}, F^{2}\right)\right\} \subseteq \Phi(\lambda+\delta)$ and $\left(\mu^{3}, F^{3}\right) \notin \Phi(\lambda+\delta)$.

Proof: Fix $\lambda \in \Lambda$ and such $|\Phi(\lambda)|>2$. Let $\left(\mu^{1}, F^{1}\right) \in \Phi(\lambda)$. Since $|\Phi(\lambda)|>2$, the arguments of Theorem 1 can be extended to show that there must be either to goods $y_{1}$ and $y_{2}$ such that $y_{j} \notin R\left(F^{1}\right)$ and $q_{j}\left(\mu^{1}, F^{1}, \lambda\right)=r_{j}(\lambda)$ for $j=\{1,2\}$; or two sets of indifference $\left(x_{a}, y_{1}\right)$ and $\left(x_{a}, y_{2}\right)$ such that $\left\{\left(x_{a}, y_{1}\right),\left(x_{b}, y_{2}\right)\right\} \in \mathcal{E}^{*}, y_{1}$ is not equal to $\mu_{a}^{1}$ or any of its direct successors in $F^{1}, y_{2}$ is not equal to $\mu_{b}^{1}$ or any of its direct successors in $F^{1}$; or one of each.

(1) Construct $\left(\mu^{2}, F^{2}\right)$ as equal to $\left(\mu^{1}, F^{1}\right)$ except that $y_{1} \in R\left(F^{2}\right)$. Similarly, $\left(\mu^{3}, F^{3}\right)$ has $y_{1}, y_{2} \in R\left(F^{2}\right)$. Now let $\left(y_{k}, y_{1}\right) \in E\left(F^{1}\right)$ and $y_{k}=\mu^{1}\left(x_{a}\right)$. Let $\delta$ be a vector of zeros with a value $\hat{\delta}<0$ in the place associated with $\xi_{a 1} \cdot q\left(\mu^{2}, F^{2}, \lambda+\delta\right)=q\left(\mu^{2}, F^{2}, \lambda+\delta\right)=$ $q\left(\mu^{2}, F^{2}, \lambda\right) . \quad q_{1}\left(\mu^{1}, F^{1}, \lambda+\delta\right)<r_{1}\left(\mu^{1}, F^{1}, \lambda+\delta\right)$. It follows that $\left(\mu^{1}, F^{1}\right) \notin \Phi(\lambda+\delta)$. Need $\left\{\left(\mu^{1}, F^{1}\right),\left(\mu^{3}, F^{3}\right)\right\} \in \Phi(\lambda+\delta)$.

(2) Construct $\left(\mu^{2}, F^{2}\right)$ as the other structure given $\left(x_{a}, y_{1}\right) \in \mathcal{E}^{*}$. Similarly $\left(\mu^{3}, F^{3}\right)$ as the other structure given $\left(x_{b}, y_{2}\right) \in \mathcal{E}^{*}$. Note that $y_{2}$ is not equal to $\mu_{a}^{2}$ or any of its direct successors in $F^{2}$. Let $\delta$ be a vector of zeros with a value $\hat{\delta}<0$ in the place associated with $\xi_{b 2}$. $\left(\mu^{3}, F^{3}\right) \notin \Phi(\lambda+\delta) . q\left(\mu^{1}, F^{1}, \lambda+\delta\right)=q\left(\mu^{2}, F^{2}, \lambda+\delta\right)=q\left(\mu^{1}, F^{1}, \lambda\right)$.

(3) Construct $\left(\mu^{2}, F^{2}\right)$ as equal to $\left(\mu^{1}, F^{1}\right)$ except that $y_{1} \in R\left(F^{2}\right)$, and construct $\left(\mu^{3}, F^{3}\right)$ as the other structure given $\left(x_{a}, y_{1}\right) \in \mathcal{E}^{*}$. Let $\delta$ be a vector of zeros with a value $\hat{\delta}<0$ in the place associated with $\xi_{a 1}$. $\left(\mu^{3}, F^{3}\right) \notin \Phi(\lambda+\delta) . q\left(\mu^{1}, F^{1}, \lambda+\delta\right)=q\left(\mu^{2}, F^{2}, \lambda+\delta\right)=q\left(\mu^{1}, F^{1}, \lambda\right)$.

The proof is completed by the observation that the only property of $\delta$ used in the proof is its direction. $\square$

Proposition (Theorem 3, Part 1): $\Lambda_{R}$ is open.

Proof: The proof is in two parts. We first prove that the set of initial conditions for which $|\phi(z)| \leq 2$ is open. We then prove that the set of initial conditions for which $Z$ is finite is open. The intersection of two open sets is open.

(1) The set of $\lambda_{0}$ for which $\left|\phi\left(z ; \lambda_{0}, S\right)\right| \leq 2$ is open. 
Given $S$, let $\Lambda_{1}$ be the set of initial conditions $\lambda_{0}$ such that $\left|\phi\left(z ; \lambda_{0}, S\right)\right| \leq 2$ for all $z \in$ $[0,1]$. Let $\tilde{\Lambda}\left[\left(\mu_{1}, F_{1}\right),\left(\mu_{2}, F_{2}\right),\left(\mu_{3}, F_{3}\right)\right]$ denote the set $\lambda_{0}$ such that there exists $z$ such that $\left|\phi\left(z ; \lambda_{0}, S\right)\right| \cap\left\{\left(\mu_{1}, F_{1}\right),\left(\mu_{2}, F_{2}\right),\left(\mu_{3}, F_{3}\right)\right\} \leq 2 . \Lambda_{1}=\cap \tilde{\Lambda}\left[\left(\mu_{1}, F_{1}\right),\left(\mu_{2}, F_{2}\right),\left(\mu_{3}, F_{3}\right)\right]$ where the intersection is over all triplets of GA structures. Each $\tilde{\Lambda}\left[\left(\mu_{1}, F_{1}\right),\left(\mu_{2}, F_{2}\right),\left(\mu_{3}, F_{3}\right)\right]$ is open by Lemma 4. Since finite intersections of open sets are open, $\Lambda_{1}$ is open.

(2) The set of $\lambda_{0}$ for which $Z$ is finite is open.

Given $\lambda_{0}$, let $Z=\left\{z \in[0, \bar{z}]|| \phi\left(z ; \lambda_{0}, S\right) \mid>1\right\}$. Given $S$, let $\Lambda_{2}$ be the set of $\lambda_{0}$ such that $Z$ is finite. Consider $\bar{\lambda}_{0} \in \Lambda_{2}$. The proof is by contradiction. Given $\varepsilon>0$, let $B_{\varepsilon}(\lambda)=$ $\left\{\lambda^{\prime} \mid d\left(\lambda, \lambda^{\prime}\right)<\varepsilon\right\}$. Suppose that for all $\varepsilon>0$, there exists $\lambda^{\prime} \in B_{\varepsilon}\left(\bar{\lambda}_{0}\right)$ such that $Z\left(\lambda^{\prime}\right)$ is infinite. Consider a sequence $\left\{\varepsilon_{n}\right\}$ converging to zero and consider a sequence $\lambda_{n}$ such that $\lambda_{n} \in B_{\varepsilon}\left(\bar{\lambda}_{0}\right)$ and $Z=\left\{z \in[0, \bar{z}]|| \phi\left(z ; \lambda_{n}, S\right) \mid>1\right\}$ is infinite. Given that there are a finite number of GA structures, there exists a subsequence $\left\{\lambda_{m}\right\} \subseteq\left\{\lambda_{n}\right\}$ and two GA structures, $\left(\mu_{1}, F_{1}\right)$ and $\left(\mu_{2}, F_{2}\right)$, such that $q\left(\mu_{1}, F_{1}, \pi\left(z ; \lambda_{m}, S\right)\right)=q\left(\mu_{2}, F_{2}, \pi\left(z ; \lambda_{m}, S\right)\right)$ at infinitely many points $z \in(0,1)$. By Lemma $3, q_{i}\left(\mu_{1}, F_{1}, \pi\left(z ; \lambda_{m}, S\right)\right)$ and $q_{i}\left(\mu_{2}, F_{2}, \pi\left(z ; \lambda_{m}, S\right)\right)$ are real analytic on $z \in(0,1)$. Given $a<0$ and $b>1$, let $\hat{S}(z):(a, b) \rightarrow \mathbb{R}^{m n+n}$ be the (component by component) analytic continuation of $S$. Given the continuity of $S$, we can choose $a, b$, and $\varepsilon_{m}$ small enough that $\lambda_{m}+\hat{S}(z)$ are contained in $\Lambda$ for all $z \in(a, b)$. Let $\hat{q}_{i}(\mu, F, z, m)=q_{i}\left(\mu, F, \lambda_{m}+\hat{S}(z)\right)$. Standard arguments establish that and $\hat{q}_{i}(\mu, F, z, m)$ is analytic and $\hat{q}_{i}(\mu, F, z, m)=q_{i}\left(\mu, F, \pi\left(z ; \lambda_{m}, S\right)\right)$ on $[0,1]$. It follows that $\hat{q}_{i}\left(\mu_{1}, F_{1}, z, m\right)=\hat{q}_{i}\left(\mu_{2}, F_{2}, z, m\right)$ at an infinite number of points in $[0,1]$. Let $U$ denote the set of intersections. It has an accumulation point in $[0,1] \subset(a, b)$. Corollary 1.2.7 in Krantz and Parks implies that $\hat{q}_{i}\left(\mu_{1}, F_{1}, z, m\right)=\hat{q}_{i}\left(\mu_{2}, F_{2}, z, m\right)$ for all $z=(a, b)$. It follows that $q\left(\mu_{1}, F_{1}, \pi\left(z ; \lambda_{m}, S\right)\right)=q\left(\mu_{2}, F_{2}, \pi\left(z ; \lambda_{m}, S\right)\right)$ for $z \in[0,1]$. The continuity of the $q(\mu, F)$ then implies that $q\left(\mu_{1}, F_{1}, \pi\left(z ; \bar{\lambda}_{0}, S\right)\right)=q\left(\mu_{2}, F_{2}, \pi\left(z ; \bar{\lambda}_{0}, S\right)\right)$. But $\bar{\lambda}_{0} \in \Lambda_{2}$. This contradiction establishes that $\Lambda_{2}$ is open.

$\Lambda_{R}=\Lambda_{1} \cap \Lambda_{2}$ each of which is open. This completes the proof. $\square$

Proposition (Theorem 3, Part 2) Let $Z^{1}\left(\lambda_{0}\right)=\{z \in[0, \bar{z}]|| \phi(z) \mid>1\}$ and let $\Lambda_{F}=$ $\left\{\lambda_{0} \mid Z\left(\lambda_{0}\right)\right.$ is finite $\}$. With $\mathrm{A}-\mathrm{C}, \Lambda_{F}$ is dense in $\Lambda_{S}$.

Proof: Given $\lambda_{0}$, let $Z=\left\{z \in[0, \bar{z}]|| \phi\left(z ; \lambda_{0}, S\right) \mid>1\right\}$. Let $\Lambda^{I} \in \Lambda_{S}$ denote the set of $\lambda_{0} \in \Lambda_{S}$ such that $Z$ is infinite. We show that given any $\lambda_{0} \in \Lambda^{I}$, we can perturb $\lambda_{0}$ and obtain a path for which $Z$ is finite.

We first prove that for each $y_{i}, \underline{p}_{i}(z)$ is real analytic on $(0,1)$ except at a finite number of points. The proof is by contradiction. Let $A=\left\{z \in(0,1) \mid \underline{p}_{i}(\lambda(z))\right.$ is not analytic at $\left.z\right\}$. Suppose 
$|A|=\infty$. Since $|A|=\infty$, there exists an accumulation point $z_{1} \in[0,1] .{ }^{9}$ Consider an arbitrary $z_{1} \in A$. There exists $\left(\mu_{1}, F_{1}\right)$ such that $\underline{p}_{i}\left(\lambda\left(z_{1}\right)\right)=q_{i}\left(\mu_{1}, F_{1}, \lambda\left(z_{1}\right)\right)$. Moreover, given any neighborhood $\Omega$ of $z_{1}$, if $\underline{p}_{i}(\lambda(z))=q_{i}\left(\mu_{1}, F_{1}, \lambda(z)\right)$ for all $z \in \Omega$ then $\underline{p}_{i}$ is analytic at $z$. It follows that there exists there a $z_{2} \in \Omega$ such that $\underline{p}_{i}\left(\lambda\left(z_{2}\right)\right) \neq q_{i}\left(\mu_{1}, F_{1}, \lambda\left(z_{2}\right)\right)$. Given the continuity of the $q_{i}(\mu, F, \lambda(z))$, this implies that there are two GA structures, $\left(\mu_{1}, F_{1}\right)$ and $\left(\mu_{2}, F_{2}\right)$, such that $q_{i}\left(\mu_{1}, F_{1}, \lambda(z)\right)=q_{i}\left(\mu_{2}, F_{2}, \lambda(z)\right)$ at some point in $\Omega$. As $z_{1}$ is arbitrary and $A$ is finite, there exist an infinite number of these intersections. Given that there are a finite number of GA structures, there are two GA structures, $\left(\mu_{3}, F_{3}\right)$ and $\left(\mu_{4}, F_{4}\right)$ such that $q_{i}\left(\mu_{3}, F_{3}, \lambda(z)\right)=q_{i}\left(\mu_{4}, F_{4}, \lambda(z)\right)$ at infinitely many points and $q_{i}\left(\mu_{3}, F_{3}, \lambda(z)\right) \neq q_{i}\left(\mu_{4}, F_{4}, \lambda(z)\right)$ at infinitely many points. Given $q_{i}\left(\mu_{3}, F_{3}, \lambda(z)\right)$ and $q_{i}\left(\mu_{4}, F_{4}, \lambda(z)\right)$ are both analytic, $q_{i}\left(\mu_{3}, F_{3}, \lambda(z)\right)=q_{i}\left(\mu_{4}, F_{4}, \lambda(z)\right)$ at infinitely many points implies $q_{i}\left(\mu_{3}, F_{3}, \lambda(z)\right)=q_{i}\left(\mu_{4}, F_{4}, \lambda(z)\right)$ at all $z \in(0,1)$. This contradiction establishes that $A$ is finite.

We return to the proof of the proposition. Suppose $\lambda_{0} \in \Lambda^{I}$. Since $\underline{p}$ is piecewise analytic, we can divide $[0,1]$ into a finite number of sub-intervals $\left\{\left[0, z_{1}\right),\left(z_{1}, z_{2}\right), \ldots\left(z_{T}, 1\right]\right\}$ such that $\underline{p}$ is analytic on each subinterval. ${ }^{10}$ Note that on each interval $\left(z_{i}, z_{i+1}\right), \phi(z)$ is constant except at a finite number of points, for if there is any GA structure $\left(\mu_{1}, F_{1}\right)$ such that $\underline{p}_{i}(\lambda(z))=$ $q_{i}\left(\mu_{1}, F_{1}, \lambda(z)\right)$ at an infinite number of points in $\left(z_{1}, z_{2}\right)$, then either $\phi(z)=\left\{\left(\mu_{1}, F_{1}\right)\right\}$ or $\left(\mu_{2}, F_{2}\right) \in \phi(z)$ and $q_{i}\left(\mu_{1}, F_{1}, \lambda(z)\right)=q_{i}\left(\mu_{2}, F_{2}, \lambda(z)\right)$ so that $\left(\mu_{1}, F_{1}\right) \in \phi(z)$ as well since both are analytic on $[0,1]$.

Let $c_{i}$ denote the number of elements of $\phi(z)$ on interval $\left(z_{i}, z_{i+1}\right)$ except possibly at a finite number of points. Given an interval in which $c_{i}>1$, we show that we can perturb $\bar{\lambda}_{0}$ and reduce $c_{i}$ by one without raising any other $c_{j}$. Given that there are finitely many intervals with values of $c_{i}>1$, a finite number of such perturbations will reduce $Z$ to a finite set.

Suppose $c_{i}>1$. We consider two cases. First, suppose that $q_{i}\left(\mu_{1}, F_{1}, \lambda(z)\right)>r_{i}(\lambda(z))$ for $y_{i} \notin R\left(F_{1}\right)$ and $z \in\left(z_{i}, z_{i+1}\right)$. According to Corollary $1,|\Phi(z)|>1$ if and only if there exists $x_{b}$ and $y_{0} \in D_{b}\left(q\left(\mu_{1}, F_{1}, \lambda\right)\right.$ such that $y_{0} \neq \mu_{b}$ and $\left(\mu_{b}, y_{0}\right) \notin E\left(F_{1}\right)$. Consider a small perturbation of $\lambda_{0}$ in which we reduce the element associated with $\xi_{b 0}$ by $\varepsilon_{b 0}$ for all such $y_{0}$. Call this perturbation $\lambda_{0}^{\prime}$ and let $\phi^{\prime}(z)$ denote the new path originating from $\lambda_{0}^{\prime}$. We choose the $\varepsilon_{b 0}$ small enough that $\lambda_{0}^{\prime} \in \Lambda_{S}$. It follows from Corollary 1 , that $\left(\mu_{2}, F_{2}\right) \notin \Phi\left(\lambda_{1}(z)\right)$ for $z \in\left(z_{i}, z_{i+1}\right)$. Note that since $q_{i}\left(\mu_{1}, F_{1}, \lambda(z)\right)=q_{i}\left(\mu_{2}, F_{2}, \lambda(z)\right)$ on $[0,1],\left(\mu_{2}, F_{2}\right) \notin \Phi\left(\lambda_{1}(z)\right)$ for all $z$. The perturbation does not raise $\phi(z)$ at any other point.

\footnotetext{
${ }^{9}$ If $z_{1}=\{0,1\}$ we will need to extend all functions as in the previous proposition to $(a, b) \supset[0,1]$ so that the accumulation point lies in an open set.

${ }^{10}$ Note that if needed we can imbed the closed interval in an open interval and extend all functions to the open interval.
} 
Second, suppose that $q_{i}\left(\mu_{1}, F_{1}, \lambda(z)\right)=r_{i}(\lambda(z))$ for some $y_{i} \notin R\left(F_{1}\right)$. In this case we consider a perturbation in which we increase the component of $\lambda_{0}$ associated with $r_{i}$. Call this perturbation $\lambda_{0}^{\prime}$ and let $\phi^{\prime}(z)$ denote the new path originating from $\lambda_{0}^{\prime}$. It is clear that $\left(\mu_{1}, F_{1}\right) \notin \Phi(\lambda(z))$ for $z \in\left(z_{i}, z_{i+1}\right)$. Similarly the perturbation does not raise $\phi$ at any other point.

This completes the proof of the proposition. $\square$

Proposition (Theorem 3, Part 3) With A-C, $\Lambda_{R}$ is dense in $\Lambda_{F}$.

Proof: Let $\pi\left(z ; \lambda_{0}\right)$ denote the path beginning at $\lambda_{0}$, let $\phi\left(z ; \lambda_{0}\right)$ denote the GAME correspondence along this path, and let $Z^{2}\left(\lambda_{0}\right)=\left\{z \in[0,1]|| \phi\left(z ; \lambda_{0}\right) \mid>2\right\}$ denote the set of points along the path at which $\phi\left(z ; \lambda_{0}\right)$ takes more than two values.

To show that $\Lambda_{R}$ is dense in $\Lambda_{F}$ we show that given an arbitrary $\bar{\lambda}_{0} \in \Lambda_{F}$ such that $Z^{2}\left(\bar{\lambda}_{0}\right)$ is not empty, we can construct a perturbation of $\bar{\lambda}_{0}, \bar{\lambda}_{0}^{\prime} \in \Lambda_{F}$, such that $Z^{2}\left(\bar{\lambda}_{0}^{\prime}\right)$ is empty.

The construction is inductive. Let $\Psi$ denote the set possible three pairs of

$$
\Psi=\left\{\left\{\left(\mu_{1}, F_{1}\right),\left(\mu_{2}, F_{2}\right),\left(\mu_{3}, F_{3}\right)\right\} \mid\left\{\left(\mu_{1}, F_{1}\right),\left(\mu_{2}, F_{2}\right),\left(\mu_{3}, F_{3}\right)\right\} \in \mathcal{G}\right\}
$$

Since $|\mathcal{G}|$ is finite, $|\Psi|=N<\infty$. At each step we choose an initial condition $\bar{\lambda}_{0}^{n} \in \Lambda_{F}$ and a radius $\delta^{n}>0$, such that $(1)$ for some $\psi_{n}$ not previously considered the set $\{z \in[0,1] \mid$ $\left.\psi_{n} \subset \phi\left(z ; \lambda_{0}\right)\right\}$ is empty for all $\lambda_{0} \in B_{\delta^{n}}\left(\bar{\lambda}_{0}^{n}\right)$; and $(2) B_{\delta^{n}}\left(\bar{\lambda}_{0}^{n}\right) \subseteq B_{\delta^{n-1}}\left(\bar{\lambda}_{0}^{n-1}\right)$, where $B_{\delta}\left(\lambda_{0}\right)=$ $\left\{\lambda \mid d\left(\lambda, \lambda_{0}\right)<\delta\right\}$. The second condition ensures that the sets $\left\{z \in[0,1] \mid \psi_{i} \subset \phi\left(z ; \lambda_{0}\right)\right\}$ is empty for all $\lambda_{0} \in B_{\delta^{n}}\left(\bar{\lambda}_{0}^{n}\right)$ and $i<n$. The iteration stops when $Z^{2}\left(\bar{\lambda}_{0}^{n}\right)$ is empty. This must happen in fewer than $N$ steps.

Initially, we choose $\bar{\lambda}_{0}^{0}=\bar{\lambda}_{0}$ and $\delta^{0}>0$ such that $B_{\delta^{0}}\left(\bar{\lambda}_{0}^{0}\right) \subset \Lambda_{S}$. This choice is possible given that $\Lambda_{S}$ is open. It will be useful below to choose $\delta^{0}$ such that the closure of $B_{\delta^{0}}\left(\bar{\lambda}_{0}^{0}\right), \bar{B}_{\delta^{0}}\left(\bar{\lambda}_{0}^{0}\right)$, is contained in $\Lambda_{S}$. Let $X=\left\{\lambda \mid \lambda=\pi\left(z, \lambda_{0}\right)\right.$ for some $\left.\lambda_{0} \in \bar{B}_{\delta^{0}}\left(\bar{\lambda}_{0}^{0}\right)\right\} . X \subset \Lambda$ is compact since $\Lambda$ is bounded. It follows that the function $f: X \rightarrow \mathbb{R}$ such that $f(\mu, F, \lambda)=\sum_{i} g_{i}(\mu, F, \lambda)$, being a continuous function from a compact metric space $X$ to a metric space $\mathbb{R}$, is uniformly continuous on $X$.

The induction step begins with $\delta^{n-1}>0, \bar{\lambda}_{0}^{n-1} \in \Lambda_{F}$, such that $B_{\delta^{n}}\left(\bar{\lambda}_{0}^{n-1}\right) \subseteq B_{\delta^{n-1}}\left(\bar{\lambda}_{0}^{n-2}\right) \subseteq$ $B_{\delta^{0}}\left(\bar{\lambda}_{0}^{0}\right)$ and $Z^{2}\left(\bar{\lambda}_{0}^{n-1}\right)$ is not empty.

Given $Z^{2}\left(\bar{\lambda}_{0}^{n-1}\right)$ is not empty, there exists $z_{1}$ such that $\left|\phi\left(z_{1} ; \bar{\lambda}_{0}^{n-1}\right)\right|>2$. Let $\hat{\lambda}=\pi\left(z_{1} ; \bar{\lambda}_{0}^{n}\right)$. It follows from Lemma 5 and the convexity of $\Lambda$ that there exists $\left\{\left(\mu^{1}, F^{1}\right),\left(\mu^{2}, F^{2}\right)\left(\mu^{3}, F^{3}\right)\right\} \equiv$ $\psi^{n} \subseteq \Phi(\hat{\lambda})$ and $l \in \mathbb{R}^{m n+n}$ such that $d(l, 0)=1$ such that for $\theta \in\left(0, \delta^{n-1}\right),\left\{\left(\mu^{1}, F^{1}\right),\left(\mu^{2}, F^{2}\right)\right\} \subseteq$ $\Phi(\hat{\lambda}+\theta l)$ and $\left(\mu^{3}, F^{3}\right) \notin \Phi(\hat{\lambda}+\theta l)$. 
Any $\theta<\delta^{n-1}$ ensures that $\hat{\lambda}+\theta l \in B_{\delta^{n-1}}\left(\bar{\lambda}_{0}^{n-1}\right)$. We must be careful, however, that we do not shift $\lambda_{0}$ so far that we create new points at which $\psi^{n} \subseteq \Phi\left(\pi\left(z, \lambda_{0}\right)\right.$. To this end, let $\hat{Z}=\left\{z \mid\left(\mu^{3}, F^{3}\right) \notin \phi\left(z ; \bar{\lambda}_{0}^{n-1}\right)\right.$ and there exist $\left.\left\{\left(\mu^{4}, F^{4}\right),\left(\mu^{5}, F^{5}\right)\right\} \subseteq \phi\left(z ; \bar{\lambda}_{0}^{n-1}\right)\right\}$ and let $\hat{\varepsilon}=\min _{z \in \hat{Z}}\left|f\left(\mu^{3}, F^{3}, \pi\left(z ; \bar{\lambda}_{0}^{n-1}\right)\right)-f\left(\mu^{1}, F^{1}, \pi\left(z ; \bar{\lambda}_{0}^{n-1}\right)\right)\right| \cdot \bar{\lambda}_{0}^{n-1} \in \Lambda_{F}$ implies $Z^{1}\left(\bar{\lambda}_{0}^{n-1}\right)$ is finite. $\hat{Z} \subseteq Z^{1}\left(\bar{\lambda}_{0}^{n-1}\right)$ implies $\hat{Z}$ is finite. $\hat{Z}$ finite implies that $\hat{\varepsilon}>0$. Since $f\left(\mu^{3}, F^{3}, \lambda\right)$ is uniformly continuous on $X$, there exists $\delta_{1}$ such that $d\left(\lambda, \lambda^{\prime}\right)<\delta_{1}$ implies $d\left(f\left(\mu^{3}, F^{3}, \lambda\right), f\left(\mu^{3}, F^{3}, \lambda^{\prime}\right)\right)<\hat{\varepsilon} / 2$. Since all paths $\pi\left(z, \lambda_{0}\right)$ have the same shape, $d\left(\lambda, \lambda^{\prime}\right)<\delta_{1}$ implies $d\left(f\left(\mu^{3}, F^{3}, \pi\left(z, \bar{\lambda}_{0}^{n-1}\right)\right), f\left(\mu^{3}, F^{3}, \pi\left(z, \lambda_{0}\right)\right)\right.$ $\hat{\varepsilon} / 2$ for all $z \in[0,1]$. If we choose $\theta>0 \operatorname{such}$ that $\theta<\min \left\{\delta^{n-1}, \delta_{1}\right\}$, then $\{z \in[0,1] \mid$ $\left.\psi^{n} \subset \phi\left(z ; \bar{\lambda}_{0}^{n-1}+\theta l\right)\right\}$ is empty. We fix $\theta \in\left(0, \min \left\{\delta^{n-1}, \delta_{1}\right\}\right)$.

Lemma 4 states that the set of $\lambda_{0}$ such that $\left|\phi\left(z ; \lambda_{0}, S\right) \cap\left\{\left(\mu_{1}, F_{1}\right),\left(\mu_{2}, F_{2}\right),\left(\mu_{3}, F_{3}\right)\right\}\right| \leq 2$ for all $z \in[0,1]$ is open. Hence there exists a neighborhood of $\bar{\lambda}_{0}^{n-1}+\theta l, \Omega$, such that $\{z \in[0,1]$ $\left.\mid \psi^{n} \subset \phi\left(z ; \bar{\lambda}_{0}^{n-1}+\theta l\right)\right\}$ is empty for all $\lambda_{0} \in \Omega$ and $\Omega \subset B_{\delta^{n-1}}\left(\bar{\lambda}_{0}^{n-1}\right)$. Given Assumptions A-C, the previous proposition states that $\Lambda_{F}$ is dense in $\Lambda_{S}$ and hence there exists $\lambda_{0}^{\prime} \in \Omega \cap \Lambda_{F}$. We set $\bar{\lambda}_{0}^{n}=\lambda_{0}^{\prime}$. We choose $\delta^{n}$ such that $B_{\delta^{n}}\left(\bar{\lambda}_{0}^{n}\right) \subseteq \Omega \subseteq B_{\delta^{n-1}}\left(\bar{\lambda}_{0}^{n-1}\right)$. This complete the induction step and the proof. 


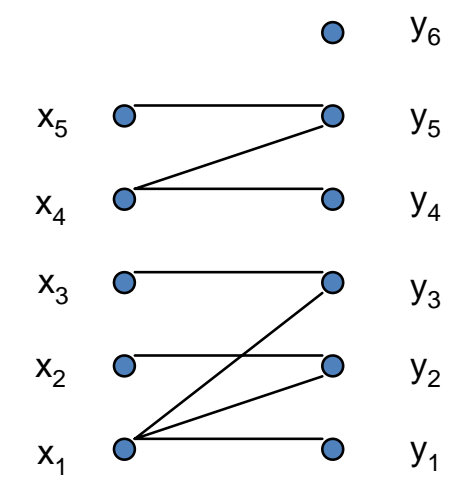

Figure 1(a)

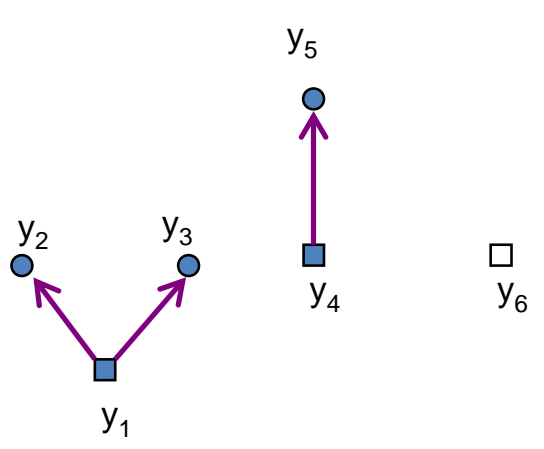

Figure 1(b) 


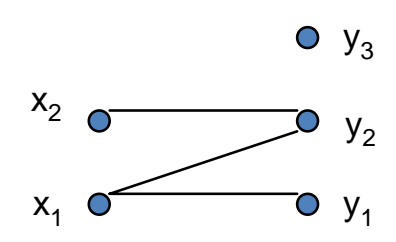

Figure 2(a)

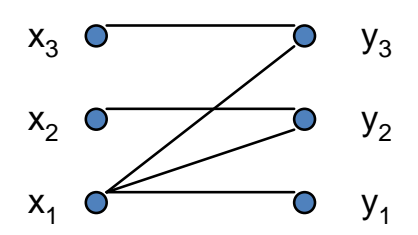

Figure 3(a)

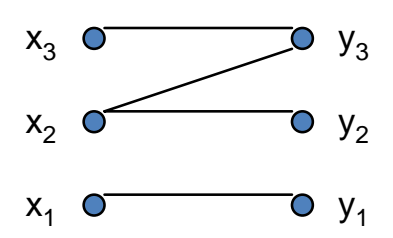

Figure 4(a)

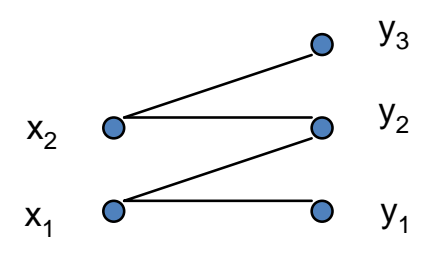

Figure 2(b)

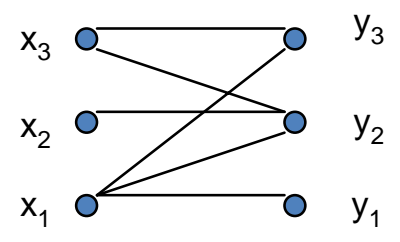

Figure 3(b)

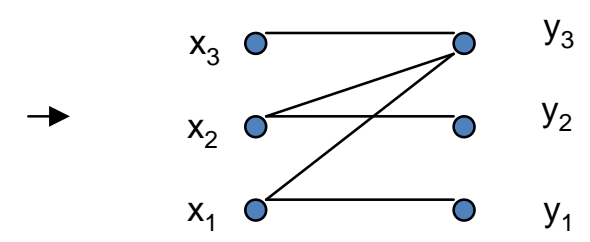

Figure 4(b)

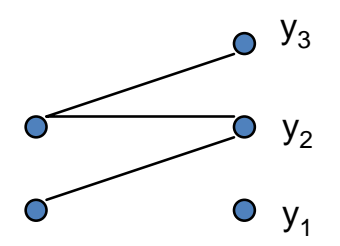

Figure 2(c)

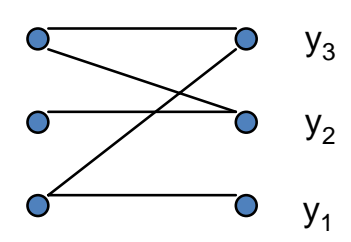

Figure 3(c)

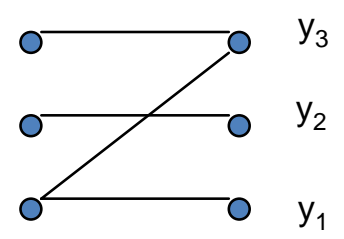

Figure 4(c) 


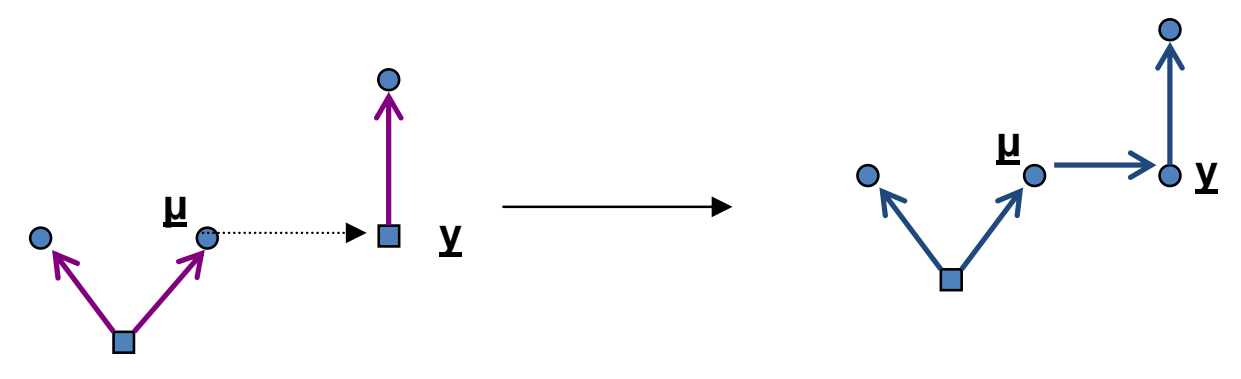

Figure 5(a)

Figure 5(b)
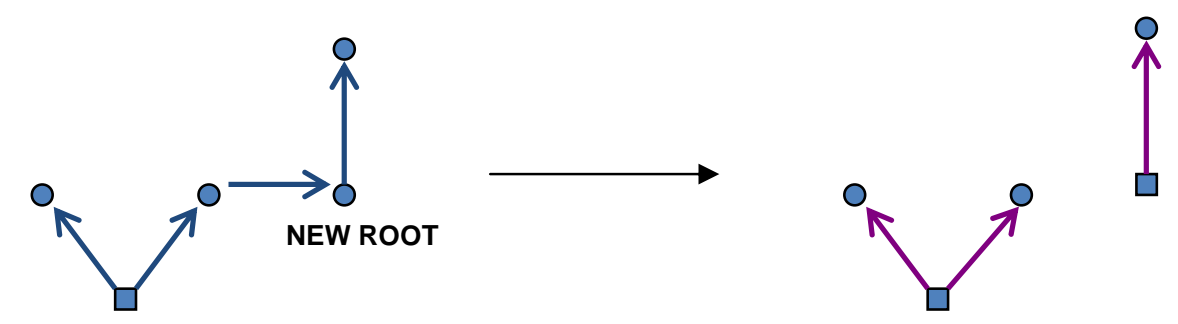

Figure 6 (a)

Figure 6(b) 


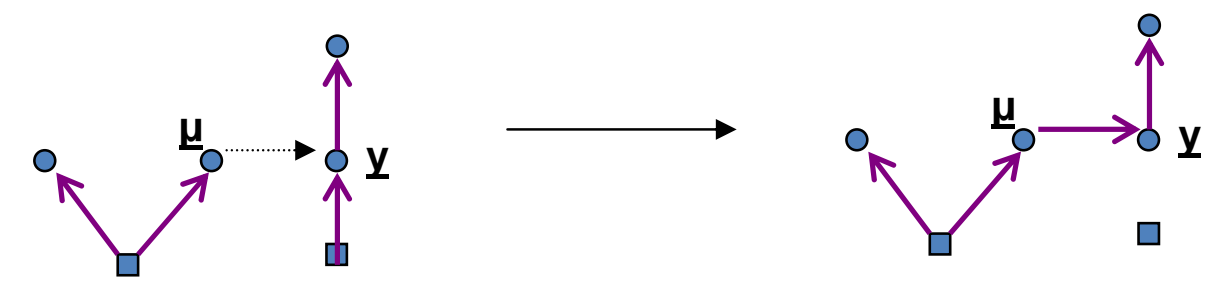

Figure 7(a)

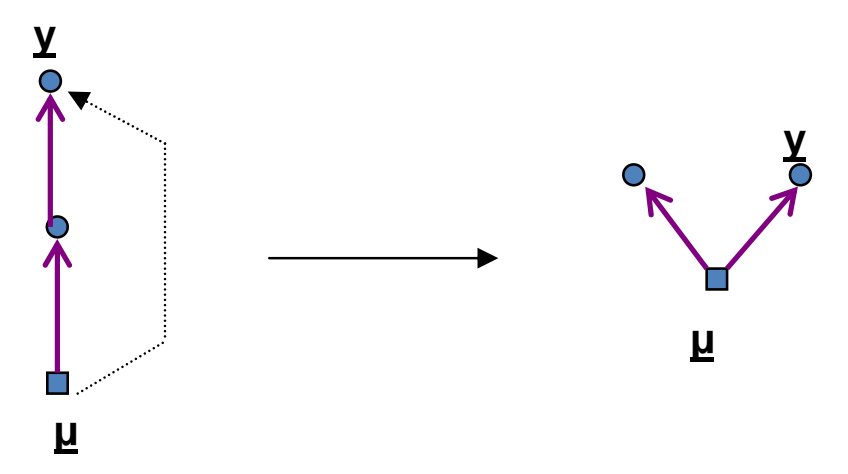

Figure 7(b) 


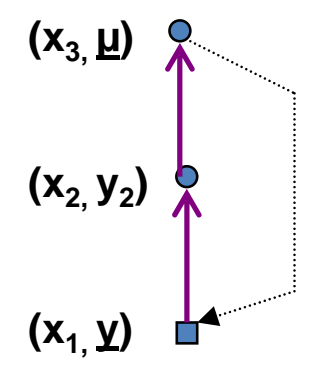

Figure 8(a)

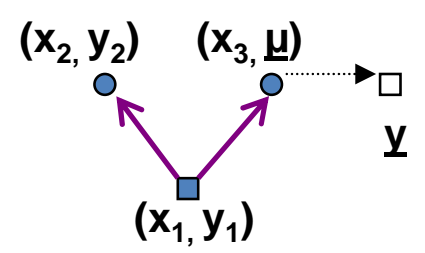

Figure 9(a)

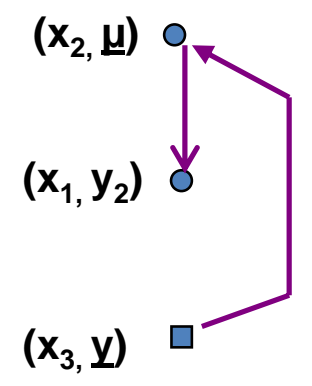

Figure 8(b)

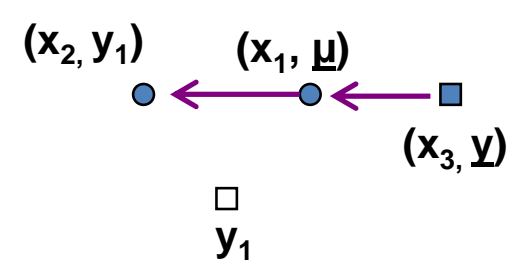

Figure 9(b) 


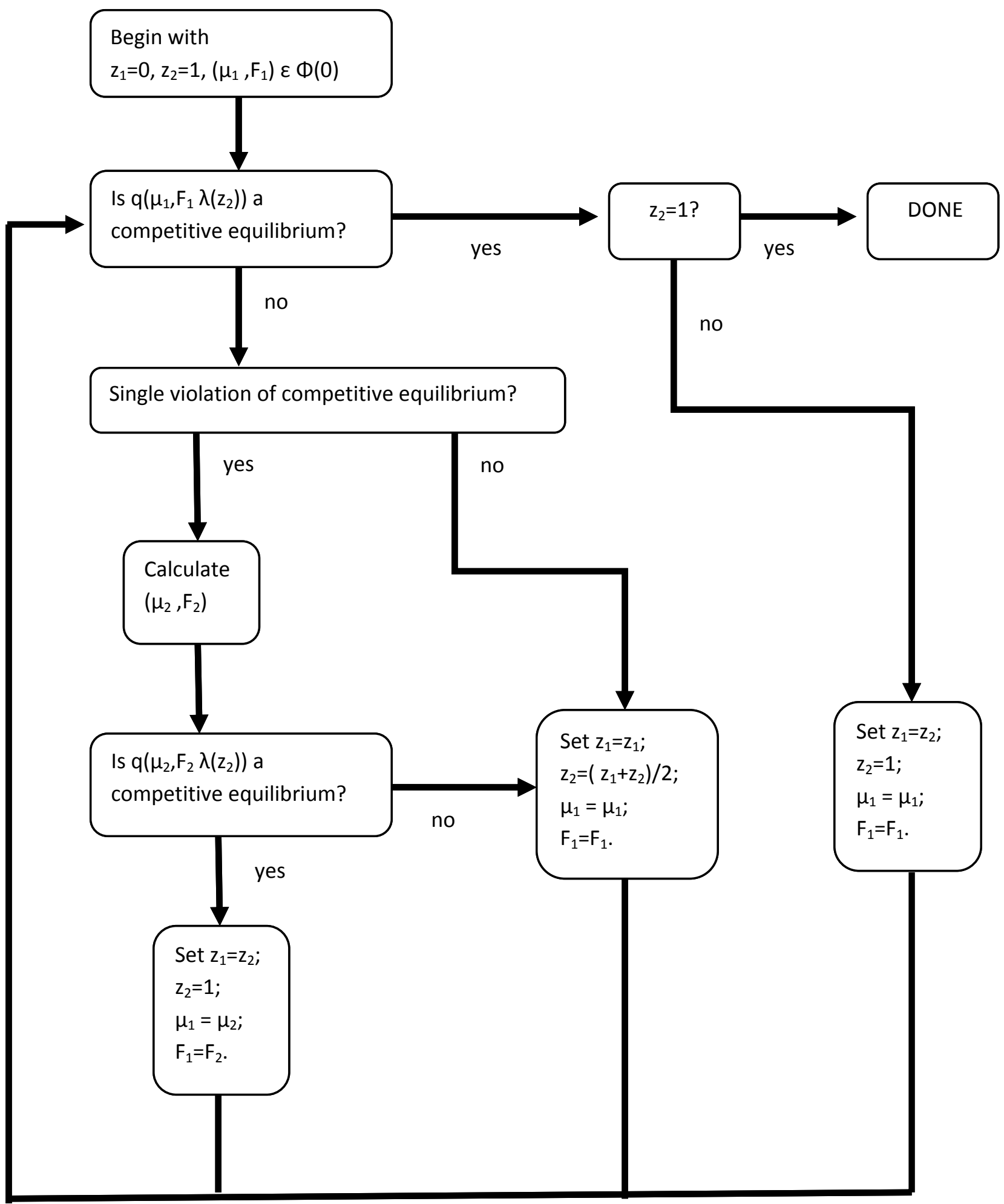

Figure 10: Flow Chart Describing Algorithm for Calculating $\Phi(1)$ from $\Phi(0)$ 